\title{
Searching for sgluons in multitop events at a center-of-mass energy of $8 \mathrm{TeV}$
}

\author{
Samuel Calvet, ${ }^{a}$ Benjamin Fuks, ${ }^{b, c}$ Philippe Gris ${ }^{a}$ and Loïc Valéry ${ }^{a}$ \\ ${ }^{a}$ Laboratoire de Physique Corpusculaire, Clermont Université, \\ Université Blaise Pascal, CNRS/IN2P3, \\ 24 avenue des Landais, F-63177 Aubière Cedex, France \\ ${ }^{b}$ Theory Division, Physics Department, CERN, \\ CH-1211 Geneva 23, Switzerland \\ ${ }^{c}$ Institut Pluridisciplinaire Hubert Curien/Département Recherches Subatomiques, \\ Université de Strasbourg/CNRS-IN2P3, \\ 23 rue du Loess, F-67037 Strasbourg, France \\ E-mail: scalvet@in2p3.fr, fuks@cern.ch, gris@clermont.in2p3.fr, \\ valery@clermont.in2p3.fr
}

ABSTRACT: Large classes of new physics theories predict the existence of new scalar states, commonly dubbed sgluons, lying in the adjoint representation of the QCD gauge group. Since these new fields are expected to decay into colored Standard Model particles, and in particular into one or two top quarks, these theories predict a possible enhancement of the hadroproduction rate associated with multitop final states. We therefore investigate multitop events produced at the Large Hadron Collider, running at a center-of-mass energy of $8 \mathrm{TeV}$, and employ those events to probe the possible existence of color adjoint scalar particles. We first construct a simplified effective field theory motivated by $R$-symmetric supersymmetric models where sgluon fields decay dominantly into top quarks. We then use this model to analyze the sensitivity of the Large Hadron Collider in both a multilepton plus jets and a single lepton plus jets channel. After having based our event selection strategy on the possible presence of two, three and four top quarks in the final state, we find that sgluon-induced new physics contributions to multitop cross sections as low as 10-100 fb can be excluded at the $95 \%$ confidence level, assuming an integrated luminosity of $20 \mathrm{fb}^{-1}$. Equivalently, sgluon masses of about $500-700 \mathrm{GeV}$ can be reached for several classes of benchmark scenarios.

Keywords: Phenomenological Models, Monte Carlo Simulations

ARXIV EPRINT: 1212.3360 


\section{Contents}

$\begin{array}{llr}1 & \text { Introduction } & 1\end{array}$

2 A simplified model for sgluon production and decays at the LHC 3

3 Technical setup for the Monte Carlo simulations $\quad 6$

$\begin{array}{lll}3.1 & \text { Objects definitions and detector simulation } & 6\end{array}$

3.2 Monte Carlo simulations of the signal and background processes 8

4 Probing sgluons via multitop events at the LHC 11

$\begin{array}{lll}4.1 & \text { Event selection for a multilepton plus jets signature } & 11\end{array}$

$\begin{array}{lll}4.2 & \text { Event selection for a single lepton plus jets signature } & 15\end{array}$

$\begin{array}{lll}\text { 4.3 LHC sensitivity to a sgluon field dominantly coupling to top quarks } & 19\end{array}$

5 Conclusion $\quad 22$

\section{Introduction}

The Standard Model of particle physics has passed for many years all experimental tests. Only the mechanism of electroweak symmetry breaking, which is currently being addressed by the general-purpose experiments ATLAS and CMS of the Large Hadron Collider (LHC), at CERN, has remained for a long time an unsolved question. The recent observation of a neutral bosonic particle compatible with a Standard-Model-like Higgs boson [1, 2], together with the imminent measurement of its properties, would then represent an impressive success of this theoretical framework. However, the mass of a fundamental scalar field is drastically affected by quantum corrections, which leads to the conceptual question of its stabilization with respect to the Planck scale lying orders of magnitude away from the weak scale. Over the last decades, large classes of alternative theories have been proposed in order to extend the Standard Model and cure this issue. Among these, weakscale supersymmetry, and in particular its minimal version, the Minimal Supersymmetric Standard Model (MSSM) [3, 4], is one of the most theoretically and experimentally studied option. It is known to solve this so-called "hierarchy problem" by introducing partners to the Standard Model degrees of freedom with opposite statistics. In addition, several other conceptual problems of the Standard Model are addressed, such as the unification of the gauge coupling strengths at high energies or the question of a viable dark matter candidate.

Experimental searches for the supersymmetric partners of the Standard Model particles are therefore among the main topics investigated at the LHC. Up to now, both the ATLAS and CMS collaborations have mainly focused on the strong production channels, since they yield larger cross sections. As a result, no traces of squarks and gluinos have been detected 
so far and the limits on the masses of the first-generation and second-generation squarks as well as those on the mass of the gluino are consequently pushed to a higher and higher scale [5-7]. Therefore, the experimental attention starts to shift towards third-generation squarks and electroweak production channels. However, all the current results may not be valid for more general supersymmetric scenarios. They are indeed either derived in the framework of the constrained version of the MSSM, where the hundreds of free parameters of the general model are reduced to a set of four parameters and one sign, or in the context of simplified models inspired by the same constrained MSSM. In contrast, there are a vast variety of non-minimal supersymmetric models which are valuable to be investigated at the LHC. In particular, some of the final state signatures predicted by these non-minimal models require dedicated phenomenological studies in order to be ready for the interpretation of the data in these theories. This is one of the scopes of the work presented in this paper.

Motivated by these arguments, we address the production and decay of color-octet massive scalar particles, also dubbed sgluons, that are predicted by several non-minimal supersymmetric models. The most considered examples are $N=1 / N=2$ hybrid supersymmetric theories [8-18] and $R$-symmetric supersymmetric theories [19-21]. In these models, the vector supermultiplet of the MSSM associated to the QCD gauge group is supplemented by an additional chiral supermultiplet lying in the adjoint representation of $\mathrm{SU}(3)$. This new supermultiplet contains, on the one hand, a two-component fermionic component which mixes with the usual gluino to form a four-component Dirac fermion. On the other hand, it also includes a color-octet complex scalar particle, i.e., a sgluon field. Let us note that color octet scalar particles also appear in vector-like confining theories [22-27] or in extra-dimensional models [28].

As all of these models are similar in the sense of the LHC signatures, we adopt the approach introduced by the LHC New Physics Working Group [29] and employ a simplified model describing a scalar octet field and its interactions with the Standard Model sector [30]. This has the major advantages to leave open the possibility of reinterpreting the results in the context of any of the original models (or even in the framework of another theory including a sgluon field) and also to avoid handling a complete model which demands a careful design of a theoretically motivated but not experimentally excluded benchmark scenario. Equivalently, rather than fixing hundreds of free parameters related to one of the new physics theories above-mentioned, we focus on a specific sector of the model, relevant for our study, that is described by a small number of couplings and masses.

We therefore start by constructing a simplified model describing the dynamics of a scalar field lying in the adjoint representation of the QCD gauge group. Motivated by complete models where loop-induced operators imply sgluon fields singly coupled to Standard Model quarks and gluons, we also include the corresponding interactions in our effective theory. Subsequently, once produced through standard strong interactions, a sgluon can then decay either to a quark pair or to a gluon pair, the latter being in general dominant for a sgluon field with a rather low mass $[10,12]$. If kinematically allowed, the same interactions ensure a possible decay to a pair of top quarks or to an associated pair comprised of one single top quark and a light jet. 
In this scenario, sgluon pair-production and decay at the LHC could lead to signatures possibly containing two, three or even four top quarks, the last two final states being expected to be largely suppressed in the context of the Standard Model. In this paper, we perform a phenomenological analysis of the sensitivity of the LHC collider, running at a center-of-mass energy $\sqrt{s}$ of $8 \mathrm{TeV}$, to the possible observation of sgluon fields through multitop signatures. This work extends our previous contribution to the 2011 Les Houches workshop [30] by including a more accurate description of the Standard Model backgrounds and a more efficient search strategy. It is also complementary to recent ATLAS and CMS investigations where sgluon-induced four-jet signatures have been considered [31-34]. In these analyses, limits on the sgluon mass up to $2 \mathrm{TeV}$ have been extracted. In our theoretical setup, we evade these bounds by imposing the sgluon field to dominantly decay to at least one top quark, as motivated by realistic supersymmetric models including a color-adjoint scalar particle. Consequently, lower sgluon masses can be possibly expected.

This paper is organized as follows. In section 2, we describe our proposal for a simplified theory modeling sgluon pair production and decay at the LHC and define benchmark scenarios for our phenomenological studies. For each benchmark point, we present the sgluon decay table and the leading-order and next-to-leading total production cross sections. Section 3 is dedicated to our technical setup for the Monte Carlo simulation of both signal and background events at $\sqrt{s}=8 \mathrm{TeV}$. A particular emphasis is put on the fast detector simulation package that we have used and in the way objects are reconstructed. In section 4, we present the details of our phenomenological analyses and the associated results. Our conclusions are given in section 5 .

\section{A simplified model for sgluon production and decays at the LHC}

In order to investigate sgluon production and decay at the LHC, we construct a simplified model describing sgluon interactions with the Standard Model fields. To this aim, we follow the approach of ref. [29] and extend the Standard Model in a minimal way by supplementing to its particle content one massive real scalar field $\sigma$ lying in the adjoint representation of the QCD gauge group. Its kinetic and mass terms are standard and can be expressed in terms of the QCD covariant derivative taken in the adjoint representation,

$$
\mathcal{L}_{\text {kin }}=\frac{1}{2} D_{\mu} \sigma^{a} D^{\mu} \sigma_{a}-\frac{1}{2} m_{\sigma}^{2} \sigma^{a} \sigma_{a} \quad \text { with } \quad D_{\mu} \sigma^{a}=\partial_{\mu} \sigma^{a}+g_{s} f_{b c}^{a} G_{\mu}^{b} \sigma^{c}
$$

where adjoint gauge indices are explicitly indicated. In the expressions above, we have introduced the strong coupling constant $g_{s}$, the antisymmetric structure constants of SU(3) $f_{b c}{ }^{a}$, the gluon field $G_{\mu}^{b}$ and the sgluon mass $m_{\sigma}$.

These interactions do not include single sgluon couplings to Standard Model fields. However, the presence of additional particles in general induces, in complete theories, loop diagrams leading to effective operators describing the coupling of a single sgluon field to up-type $(u)$ and down-type $(d)$ quark pairs, as well as to gluon pairs. In our theoretical framework, those interactions are described by the effective Lagrangian

$$
\mathcal{L}_{\text {eff }}=\sigma^{a} \bar{d} T_{a}\left[a_{d}^{L} P_{L}+a_{d}^{R} P_{R}\right] d+\sigma^{a} \bar{u} T_{a}\left[a_{u}^{L} P_{L}+a_{u}^{R} P_{R}\right] u+a_{g} d_{a}^{b c} \sigma^{a} G_{\mu \nu b} G^{\mu \nu}{ }_{c}+\text { h.c. } .
$$


The matrices $T_{a}$ and the tensor $d_{a}{ }^{b c}$ are respectively the fundamental representation matrices and the symmetric structure constants of SU(3), the operators $P_{L}$ and $P_{R}$ are the left-handed and right-handed chirality projectors acting on spin space and $G_{\mu \nu}{ }^{a}$ is the gluon field strength tensor. We have also introduced the parameters $a_{q}^{L}$ and $a_{q}^{R}$, with $q=u, d$, to model the strengths of the interactions among left-handed and right-handed quarks $q$ and a single sgluon, respectively, as well as one single parameter $a_{g}$ for the modeling of the interactions among two gluons and one sgluon. It is important to note that the interactions included in the Lagrangian of eq. (2.2) open all the possible sgluon decays to Standard Model colored particles.

Inspecting the two Lagrangians of eq. (2.1) and eq. (2.2), one observes that our simplified theory is described by one mass parameter, the sgluon mass $m_{\sigma}$, and the effective couplings of sgluons to colored partons described by a set of four complex $3 \times 3$ matrices in flavor space $a_{d}^{L}, a_{d}^{R}, a_{u}^{L}$ and $a_{u}^{R}$, together with one real (dimensionful) number $a_{g}$. Assuming $\mathcal{O}(1)$ effective interactions, sgluon masses up to about $2 \mathrm{TeV}$ are already excluded by dijet resonance searches [31]. To evade this constraint, we choose scenarios where the sgluon field dominantly decays into final states containing at least one top quark or where its couplings to a pair of light quarks or to a pair of gluons are reduced. This choice is motivated by $R$-symmetric supersymmetric theories and by $N=1 / N=2$ hybrid supersymmetric models. In these models, interactions among a single sgluon and a pair of quarks are driven by loops of squarks and gluinos (and thus suppressed by their heavy propagators). The computation of those loops lead to non-vanishing effective couplings only if at least one of the external quarks is a top quark. Furthermore, the coupling strength $a_{g}$ is related to a dimension-five operator and is thus expected to be suppressed too.

We therefore consider two series of benchmark scenarios, refered to as scenarios of class I and class II in the following. For the first set of scenarios, sgluon particles are allowed to decay, in a universal way, to any associated pair of up-type quarks containing at least one top quark. We subsequently fix

$$
\left(a_{u}^{L}\right)^{3}{ }_{i}=\left(a_{u}^{R}\right)^{3}{ }_{i}=\left(a_{u}^{L}\right)^{i}{ }_{3}=\left(a_{u}^{R}\right)^{i}{ }_{3}=3 \cdot 10^{-3},
$$

for $i=1,2,3$ and impose that all other interactions among quarks and sgluons are vanishing. From the explicit calculations of the relevant loop diagrams in ref. [10], the choice of eq. (2.3) corresponds to a scenario where squarks and gluinos have typical masses of about $1-2 \mathrm{TeV}$ and that allows for non-minimal flavor violation in the squark sector induced by supersymmetry breaking. Concerning the parameter $a_{g}$ related to the strength of the coupling among sgluons and gluons, we choose the value

$$
a_{g}=1.5 \times 10^{-6} \mathrm{GeV}^{-1} .
$$

which arises in realistic scenarios with supersymmetric masses of the same order as above and when left-handed and right-handed squarks are almost, but not, mass-degenerate [10].

In scenarios of class II, we focus exclusively on sgluon-induced LHC signatures with four top quarks and therefore forbid flavor violation in the sgluon decays. The only nonvanishing effective interactions are thus driven by the parameters

$$
\left(a_{u}^{L}\right)^{3}{ }_{3}=\left(a_{u}^{R}\right)^{3}{ }_{3}=3 \cdot 10^{-3} \quad \text { and } \quad a_{g}=1.5 \times 10^{-6} \mathrm{GeV}^{-1} .
$$




\begin{tabular}{|c|cc|}
\hline Parameters & Scenarios of type I & Scenarios of type II \\
\hline$a_{g}$ & $1.5 \times 10^{-6} \mathrm{GeV}^{-1}$ & $1.5 \times 10^{-6} \mathrm{GeV}^{-1}$ \\
$\left(a_{u}\right)^{3}{ }_{3}$ & $3 \cdot 10^{-3}$ & $3 \cdot 10^{-3}$ \\
$\left(a_{u}\right)^{3}{ }_{1}=\left(a_{u}\right)^{1}{ }_{3}$ & $3 \cdot 10^{-3}$ & 0 \\
$\left(a_{u}\right)^{3}{ }_{2}=\left(a_{u}\right)^{2}{ }_{3}$ & $3 \cdot 10^{-3}$ & 0 \\
$m_{\sigma}$ & {$[200-1000] \mathrm{GeV}$} & {$[400-1000] \mathrm{GeV}$} \\
$m_{t}$ & $172 \mathrm{GeV}$ & $172 \mathrm{GeV}$ \\
\hline
\end{tabular}

Table 1. Non-zero input parameters for benchmark scenarios of class I (second column) and II (third column). All the Standard Model parameters but the mass of the top quark (indicated in the table) follow the conventions of ref. [35].

The values of the non-zero parameters of the Lagrangians included in eq. (2.1) and eq. (2.2) are summarized, for the two series of benchmark points, in table 1. For all the Standard Model parameters, but the top quark mass $m_{t}$ which is chosen equal to $172 \mathrm{GeV}$, we follow the conventions of ref. [35]. Moreover, the sgluon mass is kept free and allowed to vary in the range $m_{\sigma} \in[200-1000] \mathrm{GeV}$ and $m_{\sigma} \in[400-1000] \mathrm{GeV}$ for class I and class II scenarios, respectively.

A key element in the multitop analysis of sgluon production and decay at the LHC lies in the sgluon branching fraction to final states containing one or two top quarks. To study the evolution of these branching ratios with the sgluon mass, the Lagrangians of eq. (2.1) and eq. (2.2) have been implemented in the FeynRules package [35-39] and the model has been exported to the UFO format [40]. We have subsequently used the matrix-element generator MADGRAPH 5 [41] to compute all sgluon partial decay widths. We then estimate the total width and the different branching ratios into two gluons, into an associated pair of a top quark and a light quark and into two top quarks. The results are shown in table 2 for the two classes of considered scenarios.

The branching of a light sgluon of few hundreds of GeV into a top-antitop pair is, as expected, kinematically suppressed compared to the other decay channels. This also holds for scenarios of class II where the sgluon field most of the time decays into a pair of gluons when it is light. For both classes of scenarios, the branching ratio of the $t \bar{t}$ decay increases with the sgluon mass (if kinematically allowed). However, the contributions of the dijet channel to the total width also become more important so that the branching into a top-antitop pair peaks for $m_{\sigma} \sim 800 \mathrm{GeV}$ and $m_{\sigma} \sim 600 \mathrm{GeV}$ for scenarios of class I and II, respectively, and then decreases for heavier sgluons.

We also show in table 2 the leading-order (LO) sgluon production cross sections as computed by the MADGRAPH 5 program for the LHC collider running at a center-ofmass energy of $8 \mathrm{TeV}$. The presented results correspond to the convolution of the tree-level matrix elements related to the Feynman diagrams of figure 1 with the LO set of the CTEQ6 parton density fit [42] and renormalization and factorization scales fixed to the transverse mass of the produced heavy particles. As sgluon-pair production cross sections are known 


\begin{tabular}{|c|cc|ccc|cc|}
\hline Scenario & $m_{\sigma}[\mathrm{GeV}]$ & $\Gamma_{\sigma}[\mathrm{MeV}]$ & $B R(t \bar{t})$ & $B R(t j / \bar{t} j)$ & $B R(g g)$ & $\sigma_{\text {tot }}[\mathrm{fb}]$ & $K_{\mathrm{NLO}}$ \\
\hline I & 200 & 0.012 & - & $80 \%$ & $20 \%$ & 98600 & 1.6 \\
\hline I & 300 & 0.105 & - & $92.3 \%$ & $7.7 \%$ & 9802 & 1.6 \\
\hline I & \multirow{2}{*}{400} & 0.219 & $4.4 \%$ & $86.9 \%$ & $8.7 \%$ & 1625 & 1.7 \\
II & & 0.029 & $33.3 \%$ & - & $66.7 \%$ & & \\
\hline I & \multirow{2}{*}{500} & 0.350 & $9.8 \%$ & $79.5 \%$ & $10.1 \%$ & \multirow{2}{*}{358.1} & 1.8 \\
II & & 0.072 & $47.8 \%$ & - & $52.2 \%$ & & \\
\hline I & \multirow{2}{*}{600} & 0.485 & $12 \%$ & $75 \%$ & $13 \%$ & \multirow{2}{*}{94.9} & 1.8 \\
II & & 0.124 & $48 \%$ & - & $52 \%$ & & \\
\hline I & \multirow{2}{*}{700} & 0.628 & $13.2 \%$ & $70.5 \%$ & $16.3 \%$ & \multirow{2}{*}{28.4} & 1.9 \\
II & & 0.185 & $44.7 \%$ & - & $55.3 \%$ & & \multirow{2}{*}{1.0} \\
\hline I & \multirow{2}{*}{800} & 0.779 & $13.5 \%$ & $66.9 \%$ & $19.6 \%$ & \multirow{2}{*}{9.26} & 2.0 \\
II & & 0.252 & $41 \%$ & - & $59 \%$ & & \multirow{2}{*}{2.1} \\
\hline I & \multirow{2}{*}{900} & 0.943 & $13.5 \%$ & $63.4 \%$ & $23.1 \%$ & \multirow{2}{*}{3.22} & 2.2 \\
II & & 0.345 & $36.9 \%$ & - & $63.1 \%$ & & \\
\hline I & \multirow{2}{*}{1000} & 1.120 & $13.2 \%$ & $60.2 \%$ & $26.6 \%$ & \multirow{2}{*}{1.17} & $2.2 \%$ \\
II & & 0.447 & $33.2 \%$ & - & $66.8 \%$ & &
\end{tabular}

Table 2. Dependence on the sgluon mass $m_{\sigma}$ of the sgluon total width $\left(\Gamma_{\sigma}\right)$, of the different branching ratios $(B R)$ to Standard Model colored particles and of the total cross section at leading order $\left(\sigma_{\text {tot }}\right)$. The next-to-leading order $K$-factors $K_{\mathrm{NLO}}$ are also indicated, extracted from ref. [44]. In the notations of the table, the symbol $j$ stands for an up or charm quark while $g$ refers to a gluon.

at the next-to-leading order (NLO) accuracy within the MADGOLEM setup [44], we also indicate in table 2 the corresponding NLO $K$-factors. In our phenomenological analyses, signal event samples are then normalized according to the NLO results.

\section{Technical setup for the Monte Carlo simulations}

\subsection{Objects definitions and detector simulation}

Detector simulation is performed with the DeLPHES program, using the public ATLAS card [45]. Jets are hence reconstructed by means of an anti- $k_{t}$ algorithm with a radius parameter set to $R=0.4$, as provided by the FASTJET package [46]. However, jets defined with such a low radius, and in particular those with a small transverse momentum, may suffer from large bias in energy reconstruction due to the magnetic field as simulated by Delphes, which spreads out the energy within the detector. Such an effect may be described by the variable

$$
\omega=\frac{E_{T}^{(\text {reco })}-E_{T}^{(\text {truth })}}{E_{T}^{\text {(truth) }}},
$$



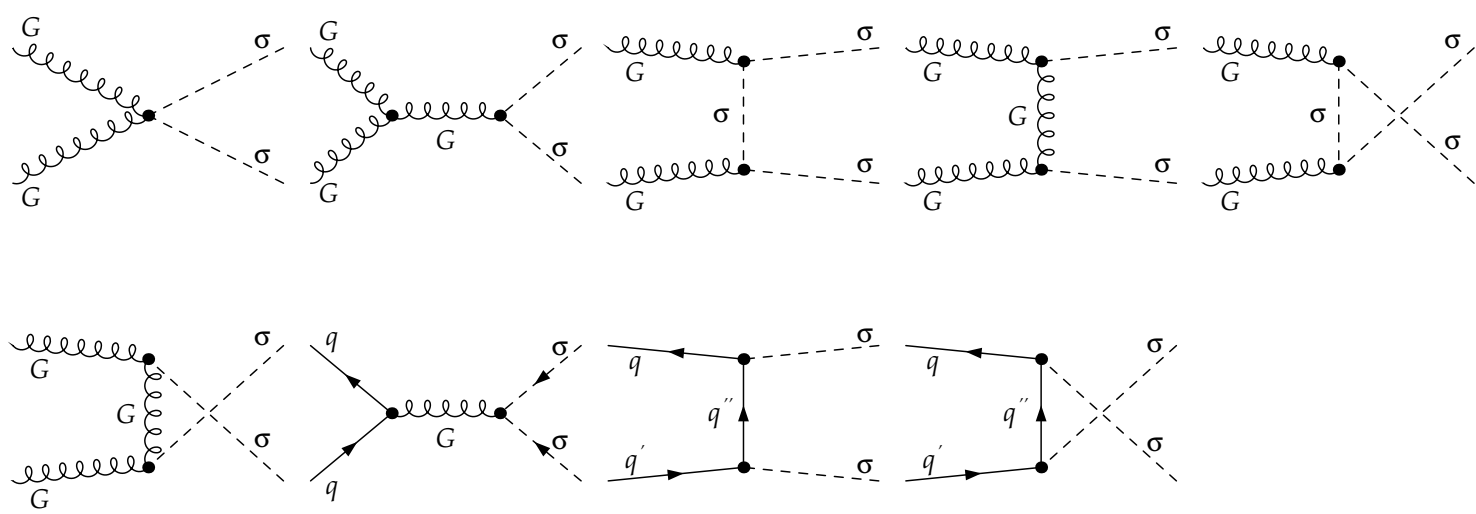

Figure 1. Tree-level Feynman diagrams associated with sgluon pair production at hadron colliders. These diagrams correspond to the interactions included in the Lagrangians of eq. (2.1) and eq. (2.2) and have been created by means of the program FEYNARTs [43].

where $E_{T}^{(\text {reco })}$ is the reconstructed jet transverse energy and $E_{T}^{(\text {truth })}$ is the true transverse jet energy defined as the jet transverse energy before detector simulation. The evolution of the $\omega$-variable with the (true) jet energy is presented on figure 2 (red squares) in the context of dijet events issued from the decay of a sequential $Z^{\prime}$-boson whose mass varies in the range $[200,1000] \mathrm{GeV}$. The energy loss reaches about $5 \%$ for jets with $E_{T}^{\text {(truth) }}=20 \mathrm{GeV}$ while it stabilizes at about $1 \%$ for jets with a transverse energy $E_{T}^{(\text {truth })}>500 \mathrm{GeV}$.

To account for this effect, an $a d$-hoc calibration is estimated from the above-mentioned dijet events. We apply on the reconstructed jet energy the correction function

$$
E_{T}^{(\mathrm{cal})}=E_{T}^{(\mathrm{reco})} \times\left[2.62 \cdot 10^{-3}-\frac{0.451 \mathrm{GeV}}{E_{T}^{(\mathrm{reco})}} \ln \frac{E_{T}^{(\text {reco })}}{\mathrm{GeV}}\right],
$$

where $E_{T}^{(\mathrm{cal})}$ is the jet transverse energy after calibration and we show the associated effects on figure 2 (blue circles). This calibration procedure allows us to recover the correct jet energy for transverse energy as low as $E_{T}^{\text {(truth) }}$ of about $40 \mathrm{GeV}$.

In our analysis, only jets with a transverse energy $E_{T}>20 \mathrm{GeV}$ (after calibration) and a pseudorapidity $|\eta|<2.5$ are retained. In addition, we estimate a $b$-tagging efficiency of about $60 \%$, together with a charm and light flavor mistagging rate of $10 \%$ and $1 \%$, respectively.

Charged leptons candidates ${ }^{1}$ are requested to have a transverse momentum $p_{T}$ larger than $20 \mathrm{GeV}$ and a pseudorapidity $|\eta|<2.47$ and $|\eta|<2.5$ for electrons and muons, respectively. We also impose two isolation criteria. First, the closest jet to an electron is removed if the angular distance $\Delta R=\sqrt{\Delta \phi^{2}+\Delta \eta^{2}} \leq 0.1$, where $\phi$ stands for the azimutal angle with respect to the beam direction. Secondly, in the case at least one jet is present within a cone of radius $R=0.4$ centered on the lepton, the lepton is removed from the event.

\footnotetext{
${ }^{1}$ By the generic terminology charged leptons, we only consider electrons and muons.
} 


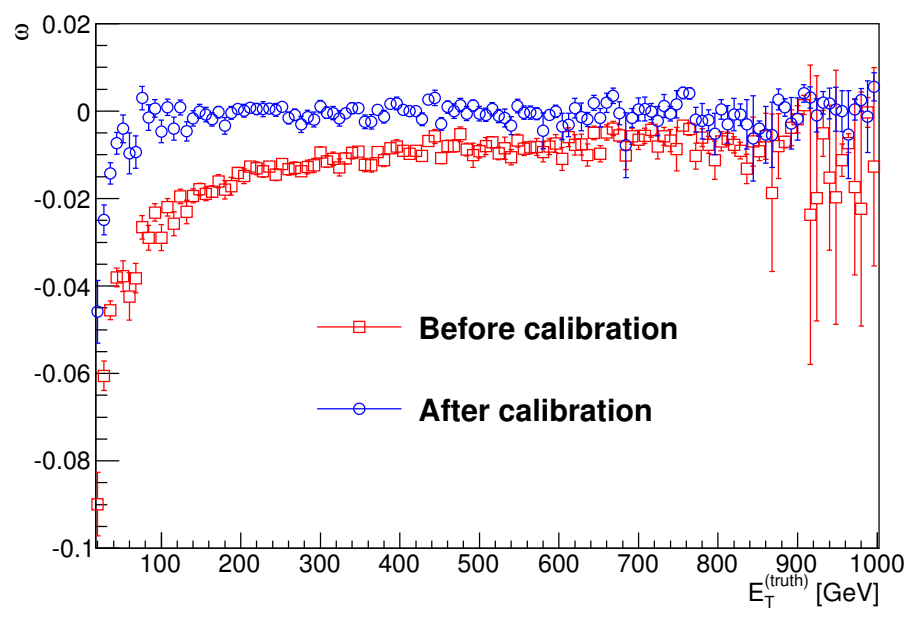

Figure 2. Evolution of the $\omega$-variable defined in eq. (3.1) with respect to the true transverse energy of the reconstructed jet before (red squares) and after (blue circles) calibration.

\subsection{Monte Carlo simulations of the signal and background processes}

We perform in this work a prospective phenomenological analysis of multitop events induced by the production of a pair of sgluon fields. We aim to estimate the LHC sensitivity to the presence of sgluons in such events by means of a phenomenological analysis employing Monte Carlo simulations. Our analysis context consists of the LHC collider running at a center-of-mass energy of $\sqrt{s}=8 \mathrm{TeV}$ and we normalize our event samples to an integrated luminosity of $20 \mathrm{fb}^{-1}$, which corresponds to the expectations for the end of the $2012 \mathrm{LHC}$ run $[47]$.

The hard scattering processes related to both the signal and the different sources of background have been described with the matrix-element generator MADGRAPH 5 [41]. Using the QCD factorization theorem, the matrix elements are convolved with the leading order set of the CTEQ6 parton density fit [42] and the renormalization and factorization scales are fixed to the transverse mass of the produced heavy particles. Parton-level events are then integrated into a full hadronic environment by matching the hard scattering matrix elements to the full parton showering and hadronization infrastructure as provided by the Pythia 6 package [48]. We have then included a fast detector simulation as performed by the program DeLPhes 1.9 [45], using, as stated above, the publicly available ATLAS detector card.

The procedure above is however known not to provide an accurate description of the kinematical properties of jets due to an underestimation of the hard emission effects by parton showering algorithms which only correctly model jets in the soft and collinear limit. In contrast, matrix elements properly describe additional hard and widely separated radiation, but are known to break down in the soft and collinear kinematical regions. Therefore, we allow for the matrix elements related to our background samples to contain zero, one, two, etc, additional hard jets. The resulting events are then merged following the Mangano (MLM) procedure [49] as implemented in the MADEvENT program [50]. 
In this setup, two parton-level selection criteria are imposed. Firstly, the squared jet measure $k_{T}^{2}=\min \left(p_{T i}^{2}, p_{T j}^{2}\right) R_{i j}$ related to two final state partons is asked to be larger than a process-dependent value $\left(k_{T}^{\min }\right)^{2}$, where $p_{T i}$ and $p_{T j}$ are the transverse momenta of the two partons and $R_{i j}$ their angular distance in the $(\eta, \phi)$ plane. Secondly, the jet measure related to a final-state and an initial-state parton is defined as the transverse momentum of the final-state parton and is asked to be larger than a process-dependent value $p_{T}^{\min }$. The events are then passed to PYTHIA and jets are reconstructed using the FASTJET program [46], making use of a $k_{T}$-jet algorithm with a cut-off scale $Q^{\mathrm{m}}$. An event is kept only if, for each of the reconstructed jets in the event, the jet measure between the jet and the parton which it is originating from is smaller than $Q^{\mathrm{m}}$. In order to maintain the full inclusiveness of the merged sample, extra jets with respect to the original number of partons are allowed in the sample with the highest multiplicity. The merging scale $Q^{\mathrm{m}}$ is process-dependent and chosen in such a way that the differential jet rate distributions of the merged samples are smooth. The values chosen for the parton-level selections, the maximum number of included hard emissions and the merging scale are given in table 3 for the various background processes. Concerning the signal, no merging with matrix elements of a higher multiplicity has been performed as the lowest order subprocess already contains many hard jets.

Focusing first on the signal, we generate, for each scenario and each sgluon mass (see table 1 and table 2), three event samples according to the top multiplicity of the final state, the latter being taken equal to two, three and four top quarks, respectively. Our event sample normalization includes the NLO $K$-factor presented in table 2 . Turning to the background, we are planning to require, in our analysis, the presence of at least one isolated charged lepton. We therefore only simulate the Standard Model background processes presented in table 3, which also contains, in addition to the generation parameters above-mentioned, the cross sections employed for the normalization of the samples and the associated numbers of generated events.

We first address the simulation of weak gauge boson production in association with jets. We have merged event samples containing up to four additional hard jets and the gauge bosons are enforced to decay leptonically. Parton-level events having also been allowed to contain tau leptons, we make use of the TAUOLA program [51] in order to handle their decays. We have reweighted the events according to the next-to-next-to-leading order (NNLO) cross sections as computed by the FEwZ package [52-54] with the recent set of parton densities CT10 provided by the CTEQ collaboration [55]. Virtual photon contributions are included where relevant, together with a parton-level selection based on the dilepton invariant mass $m_{\ell \ell}>50 \mathrm{GeV}$.

We now turn to background events related to top quark production. We generate two distinct $t \bar{t}$ samples, one associated to the semileptonic decay of the $t \bar{t}$ pair and one related to its dileptonic decay. Our merging procedure includes matrix elements containing up to two additional jets and we have reweighted all the events according to the production cross section at the NLO accuracy, including genuine NNLO contributions, as predicted by the HAтHOR program $[56,57]$. Single top event generation has been split into the generation of three different inclusive samples, following the usual parton-level distinction between $s$ channel diagrams where the top quark is produced in association with a $b$ quark, $t$-channel 


\begin{tabular}{|l|cc|cc|cc|}
\hline Process & $k_{T}^{\mathrm{min}}[\mathrm{GeV}]$ & $p_{T}^{\mathrm{min}}[\mathrm{GeV}]$ & $n$ & $Q^{\mathrm{m}}[\mathrm{GeV}]$ & $\sigma[\mathrm{pb}]$ & $N$ \\
\hline$W(\rightarrow 1 \ell)+$ jets & 10 & 10 & 4 & 20 & 35678 & $2 \cdot 10^{10}$ \\
$\gamma^{*} / Z(\rightarrow 2 \ell)+$ jets & 10 & 10 & 4 & 20 & 3460 & $4 \cdot 10^{6}$ \\
\hline$t \bar{t}(\rightarrow 1 \ell)+$ jets & 20 & 20 & 2 & 30 & 112. & $9 \cdot 10^{6}$ \\
$t \bar{t}(\rightarrow 2 \ell)+$ jets & 20 & 20 & 2 & 30 & 27.2 & $3 \cdot 10^{6}$ \\
\hline$t / \bar{t}+$ jets $[t$, incl. $]$ & - & - & 0 & - & 28.4 & $4 \cdot 10^{6}$ \\
$t / \bar{t}+$ jets $[t W$, incl. $]$ & - & - & 0 & - & 12.1 & $2 \cdot 10^{6}$ \\
$t / \bar{t}+$ jets $[s$, incl. $]$ & - & - & 0 & - & 1.81 & $8 \cdot 10^{5}$ \\
\hline$W W(\rightarrow 1 \ell)+$ jets & 10 & 10 & 2 & 20 & 24.3 & $3 \cdot 10^{6}$ \\
$W W(\rightarrow 2 \ell)+$ jets & 10 & 10 & 2 & 20 & 5.87 & $8 \cdot 10^{5}$ \\
$W Z(\rightarrow 1 \ell)+$ jets & 10 & 10 & 2 & 20 & 6.47 & $2 \cdot 10^{5}$ \\
$W Z(\rightarrow 2 \ell)+$ jets & 10 & 10 & 2 & 20 & 1.58 & $2 \cdot 10^{5}$ \\
$W Z(\rightarrow 3 \ell)+$ jets & 10 & 10 & 2 & 20 & 0.76 & $7 \cdot 10^{4}$ \\
$Z Z(\rightarrow 4 \ell)+$ jets & 10 & 10 & 2 & 20 & 0.17 & $4 \cdot 10^{4}$ \\
$Z Z(\rightarrow 2 \ell)+$ jets & 10 & 10 & 2 & 20 & 1.50 & $4 \cdot 10^{4}$ \\
\hline$t \bar{t} W+$ jets [incl.] & 10 & 10 & 2 & 20 & 0.25 & $3 \cdot 10^{4}$ \\
$t \bar{t} Z+$ jets [incl. $]$ & 10 & 10 & 2 & 20 & 0.21 & $5 \cdot 10^{4}$ \\
$t \bar{t} W W+$ jets [incl. $]$ & 10 & 10 & 2 & 20 & 0.013 & $2 \cdot 10^{3}$ \\
$t \bar{t} t \bar{t}+$ jets [incl. $]$ & - & - & 0 & - & $7 \cdot 10^{-4}$ & $10^{3}$ \\
\hline
\end{tabular}

Table 3. We present the simulated background processes, together with the set of applied parton-level selection criteria $\left(k_{T}^{\min }\right.$ and $\left.p_{T}^{\min }\right)$, the number of allowed extra hard emissions at the matrix-element level $(n)$ and the matching scale $\left(Q^{\mathrm{m}}\right)$. The numerical values employed for the cross sections $(\sigma)$ are also given, together with the number of generated events $(N)$. For each of the background process, the final state contains at least one lepton $\ell$, where $\ell$ stands equivalently for electrons, muons, leptonic and hadronic taus. The notations incl. indicates that the associated samples are inclusive in the decays. We refer to the text for a more detailed description and for information on the adopted values for the cross sections.

diagrams where the top quark is produced in association with a light jet, and $t W$ diagrams describing the associated production of a top quark and a $W$-boson. In order to maintain this distinction non-ambiguous, the MLM merging procedure has not been applied. This avoids a possible double counting over the three channels since specific diagrams with extra radiation could in principle belong to several of the categories, even though the kinematical regimes are different. The events are then reweighted according to NLO cross sections including genuine NNLO contributions [58-61].

Concerning diboson production, we have performed a merging of matrix elements including up to two additional hard jets and have normalized the cross sections to the NLO accuracy as provided by the MCFM package [62,63]. In our setup, we have included virtual 
photon contributions where relevant and consequently imposed a selection on the dilepton invariant-mass of $m_{\ell \ell}>50 \mathrm{GeV}$.

Rare Standard Model processes where a top-antitop pair is produced in association with one or two gauge bosons are also considered. We generate inclusive event samples for the $t \bar{t} W, t \bar{t} Z$ and $t \bar{t} W W$ processes and perform MLM-merging of matrix elements containing up to two extra jets. We normalize the samples to the NLO cross sections as predicted by MCFM [64] for the $t \bar{t} W$ and $t \bar{t} Z$ processes and to the LO results as returned by MADGraph for the $t \bar{t} W W$ process. Finally, four-top production is also simulated but no merging is performed since the lowest order matrix-element already contains many hard jets. This event sample is normalized to the LO accuracy.

\section{Probing sgluons via multitop events at the LHC}

In this paper, we wish to estimate the LHC sensitivity to the search for multitop events originating from sgluon decays. According to the two classes of scenarios under consideration (see table 1 and table 2), sgluon pair production and decay lead to three topologies comprised of two top quarks and two light jets $(t j t j)$, three top quarks and one light jet $(t j t t)$ and four top quarks $(t t t t)$, the symbol $t$ denoting equivalently a top and an antitop quark and the symbol $j$ a light jet or $b$-jet. The first two signatures are only considered assuming scenarios of class I, while four-top final states are produced in the context of both classes of scenarios. The corresponding final states are thus characterized by a large number of hard jets (between four and twelve) with an important heavy-flavor content arising from top decays. The large branching ratio associated with top quark hadronic decays would naively encourage us to search for sgluon pairs in fully hadronic final states. This analysis would however suffer from an overwhelming multijet background whose a correct estimation requires data-driven methods. To optimize the sensitivity of our search, we therefore restrict ourselves to leptonic final states. We design two analyses, one dedicated to events containing exactly one single lepton and another one to events with at least two leptons.

\subsection{Event selection for a multilepton plus jets signature}

Events are preselected with the requirement that they contain exactly two (for the $t j t j$ topology) or at least two charged leptons (for the $t j t t$ and $t t t t$ topologies) with a transverse momentum $p_{T}^{\ell}>20 \mathrm{GeV}$. The invariant mass of the pair comprised of the two leading leptons $m_{\ell \ell}$ is also requested to be larger than $50 \mathrm{GeV}$ in order to be compatible with the parton-level selection criterion of section 3.2. At this stage, the total Standard Model background contains a large part of Drell-Yan lepton pair events $(98.7 \%$ and $98.2 \%$ for the $t j t j$ and $t j t t / t t t t$ topologies, respectively). To reduce this background, a selection on the missing transverse energy $\mathbb{E}_{T}$, defined as

$$
\mathbb{E}_{T}=\left\|\sum_{\text {visible particles }} \vec{p}_{T}\right\|,
$$



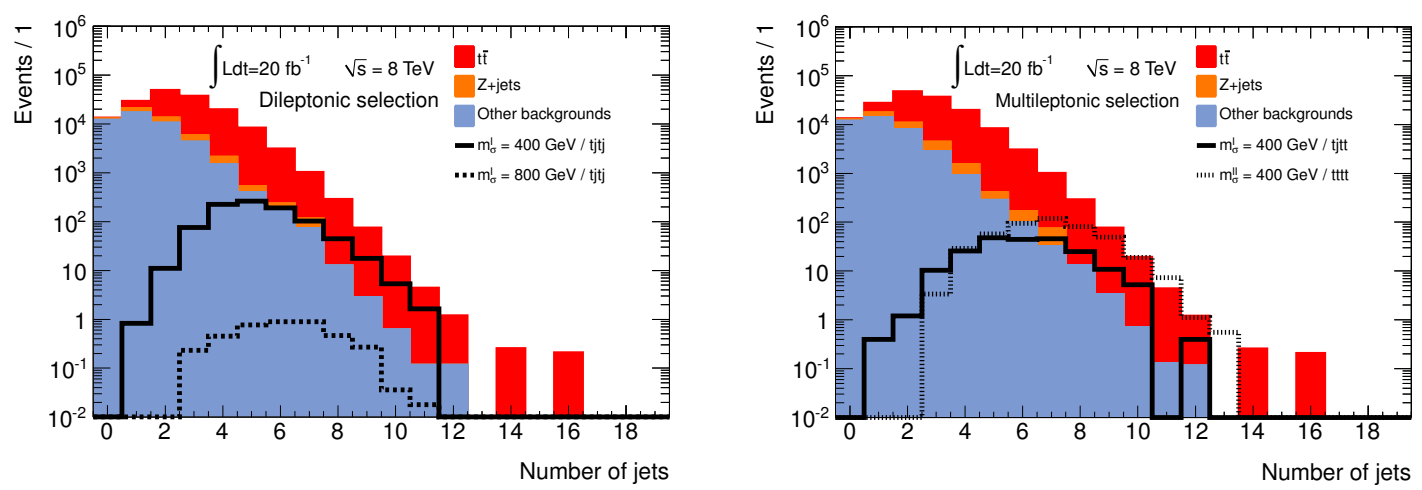

Figure 3. Jet multiplicity distribution after selecting events with exactly (left panel) or at least (right panel) two leptons, missing transverse energy $\mathbb{E}_{T}>40 \mathrm{GeV}$ and a dilepton invariant-mass $m_{\ell \ell}>50 \mathrm{GeV}$. Contributions arising from top-antitop (red) and Drell-Yan production (orange) are factorized from the rest of the background (blue) and signal distributions for the $t j t j$ signature (left panel) and for both the $t j t t$ and $t t t t$ channels (right panel) are indicated by plain and dashed curves.

is applied. By requiring $\mathbb{E}_{T}>40 \mathrm{GeV}$, we take advantage of the fact that Drell-Yan events are characterized by a lack of missing energy whereas the neutrinos arising from leptonic top decays ensure signal events to contain a sensible quantity of missing energy.

Jets present in sgluon-pair events originate mainly from the hadronization of $b$-quarks and light quarks issued from top decays. In contrast, the hadronic activity of background events consists dominantly of initial-state radiation that leads to a lower jet multiplicity (see figure 3). To maintain simultaneously a good sensitivity to the sgluon signal while discarding a substantial part of the background, the presence of at least three, four and five jets with $p_{T}^{j}>25 \mathrm{GeV}$ is demanded for the $t j t j$, $t j t t$ and $t t t t$ final states, respectively. In addition, we benefit from the presence of jets arising from the fragmentation of long-lived $b$-quarks in sgluon-induced events, requiring a minimum number of one, two and three $b$-tagged jets for the $t j t j, t j t t$ and $t t t t$ topologies, respectively.

After applying the above-mentioned selections to the preselected dileptonic events, the selection efficiencies for the signal are found to range from $15 \%$ to $50 \%$ for a sgluon mass of $m_{\sigma}=400 \mathrm{GeV}$ and from $25 \%$ to $60 \%$ for $m_{\sigma}=800 \mathrm{GeV}$ for the three search channels (derived from table 4). The expected Standard Model background, which has been divided by a factor of about 400, 3000 and 100000 for the $t j t j$, tjtt and $t t t t$ search strategies, respectively, is now largely comprised of Drell-Yan and top-antitop (plus possibly one or two additional gauge bosons) events. To further reduce it, we apply a specific selection on the fraction of dileptonic events and only retain those where the leptons have the same electric charge. The signal efficiency of such a criterion is of about $50 \%$ while only $10 \%$ and $20 \%$ of the background events survive in the context of the $t j t j$ and $t j t t / t t t t$ topologies.

The numbers of events selected at each step of the analysis are indicated in table 4 for two representative benchmark scenarios with sgluon masses of $400 \mathrm{GeV}$ and $800 \mathrm{GeV}$, as well as for the background. It turns out that after all selection criteria, the background is dominated by top-antitop events for the $t j t j$ and $t j t t$ search channels and by events related 


\begin{tabular}{|c|ccc|}
\hline Selections & \multicolumn{3}{|c|}{$t j$ tj channel } \\
& $m_{\sigma}^{I}=400 \mathrm{GeV}$ & $m_{\sigma}^{I}=800 \mathrm{GeV}$ & Backgrounds \\
\hline$N_{\ell}=2$ with $p_{T}^{\ell} \geq 20 \mathrm{GeV}$ & $(1.26 \pm 0.02) \cdot 10^{3}$ & $4.86 \pm 0.30$ & $(1.721 \pm 0.002) \cdot 10^{7}$ \\
$m_{\ell \ell} \geq 50 \mathrm{GeV}$ & $(1.15 \pm 0.02) \cdot 10^{3}$ & $4.49 \pm 0.28$ & $(1.716 \pm 0.002) \cdot 10^{7}$ \\
$\mathbb{E}_{T} \geq 40 \mathrm{GeV}$ & $(9.38 \pm 0.20) \cdot 10^{2}$ & $4.04 \pm 0.27$ & $(1.549 \pm 0.004) \cdot 10^{5}$ \\
$N_{j} \geq 3$ with $p_{T}^{j} \geq 25 \mathrm{GeV}$ & $(9.18 \pm 0.19) \cdot 10^{2}$ & $4.04 \pm 0.27$ & $(5.693 \pm 0.020) \cdot 10^{4}$ \\
$N_{b} \geq 1$ & $(6.05 \pm 0.16) \cdot 10^{2}$ & $2.80 \pm 0.22$ & $(4.089 \pm 0.011) \cdot 10^{4}$ \\
Same sign dilepton & $(2.81 \pm 0.11) \cdot 10^{2}$ & $1.06 \pm 0.14$ & $(4.191 \pm 0.035) \cdot 10^{3}$ \\
\hline
\end{tabular}

\begin{tabular}{|c|ccc|}
\hline Selections & \multicolumn{3}{|c|}{$t j t t$ channel } \\
& $m_{\sigma}^{I}=400 \mathrm{GeV}$ & $m_{\sigma}^{I}=800 \mathrm{GeV}$ & Backgrounds \\
\hline$N_{\ell} \geq 2$ with $p_{T}^{\ell} \geq 20 \mathrm{GeV}$ & $(2.89 \pm 0.11) \cdot 10^{2}$ & $4.71 \pm 0.17$ & $(1.722 \pm 0.002) \cdot 10^{7}$ \\
$m_{\ell \ell} \geq 50 \mathrm{GeV}$ & $(2.63 \pm 0.10) \cdot 10^{2}$ & $4.44 \pm 0.17$ & $(1.717 \pm 0.002) \cdot 10^{7}$ \\
$\not_{T} \geq 40 \mathrm{GeV}$ & $(2.17 \pm 0.09) \cdot 10^{2}$ & $4.12 \pm 0.16$ & $(1.598 \pm 0.004) \cdot 10^{5}$ \\
$N_{j} \geq 4$ with $p_{T}^{j} \geq 25 \mathrm{GeV}$ & $(1.97 \pm 0.09) \cdot 10^{2}$ & $4.03 \pm 0.16$ & $(2.375 \pm 0.012) \cdot 10^{4}$ \\
$N_{b} \geq 2$ & $83 \pm 6$ & $1.89 \pm 0.11$ & $(5.950 \pm 0.040) \cdot 10^{3}$ \\
Same sign dilepton & $36 \pm 4$ & $0.77 \pm 0.07$ & $(2.860 \pm 0.080) \cdot 10^{2}$ \\
\hline
\end{tabular}

\begin{tabular}{|c|ccc|}
\hline Selections & \multicolumn{3}{|c|}{$t t t$ channel } \\
& $m_{\sigma}^{I}=400 \mathrm{GeV}$ & $m_{\sigma}^{I I}=800 \mathrm{GeV}$ & Backgrounds \\
\hline$N_{\ell} \geq 2$ with $p_{T}^{\ell} \geq 20 \mathrm{GeV}$ & $11.33 \pm 0.33$ & $7.90 \pm 0.24$ & $(1.722 \pm 0.002) \cdot 10^{7}$ \\
$m_{\ell \ell} \geq 50 \mathrm{GeV}$ & $10.42 \pm 0.32$ & $7.56 \pm 0.22$ & $(1.717 \pm 0.002) \cdot 10^{7}$ \\
$\notin_{T} \geq 40 \mathrm{GeV}$ & $8.78 \pm 0.29$ & $7.03 \pm 0.21$ & $(1.598 \pm 0.004) \cdot 10^{5}$ \\
$N_{j} \geq 5$ with $p_{T}^{j} \geq 25 \mathrm{GeV}$ & $7.50 \pm 0.27$ & $6.60 \pm 0.20$ & $(8.11 \pm 0.06) \cdot 10^{3}$ \\
$N_{b} \geq 3$ & $1.61 \pm 0.13$ & $1.93 \pm 0.11$ & $(1.88 \pm 0.06) \cdot 10^{2}$ \\
Same sign dilepton & $0.69 \pm 0.08$ & $0.82 \pm 0.07$ & $10.3 \pm 1.5$ \\
\hline
\end{tabular}

Table 4. Flow-charts related to the selection strategy for the $t j t j$ (upper panel), $t j t t$ (middle panel) and $t t t t$ (lower panel) topologies in the case of a multilepton analysis. We present the remaining number of events, together with their associated statistical uncertainties, after each of the selection criteria in the context of the LHC collider running at a center-of-mass energy of $\sqrt{s}=8 \mathrm{TeV}$ and for an integrated luminosity of $20 \mathrm{fb}^{-1}$. For the $t j t j$ and $t j t t$ channels, signal events are given for scenarios of class I and for a sgluon mass of $m_{\sigma}^{I}=400$ (800) GeV in the second (third) column of the tables. Concerning the tttt channel, we present in the second (third) column of the table the evolution of the number of signal events for a scenario of class I (II) with a sgluon mass of $m_{\sigma}^{I}=400 \mathrm{GeV}\left(m_{\sigma}^{I I}=800 \mathrm{GeV}\right)$ after applying each of the selections. The associated numbers of background events are shown in the fourth column of the tables. For a detailed description of each of the selections, we refer to the text. 

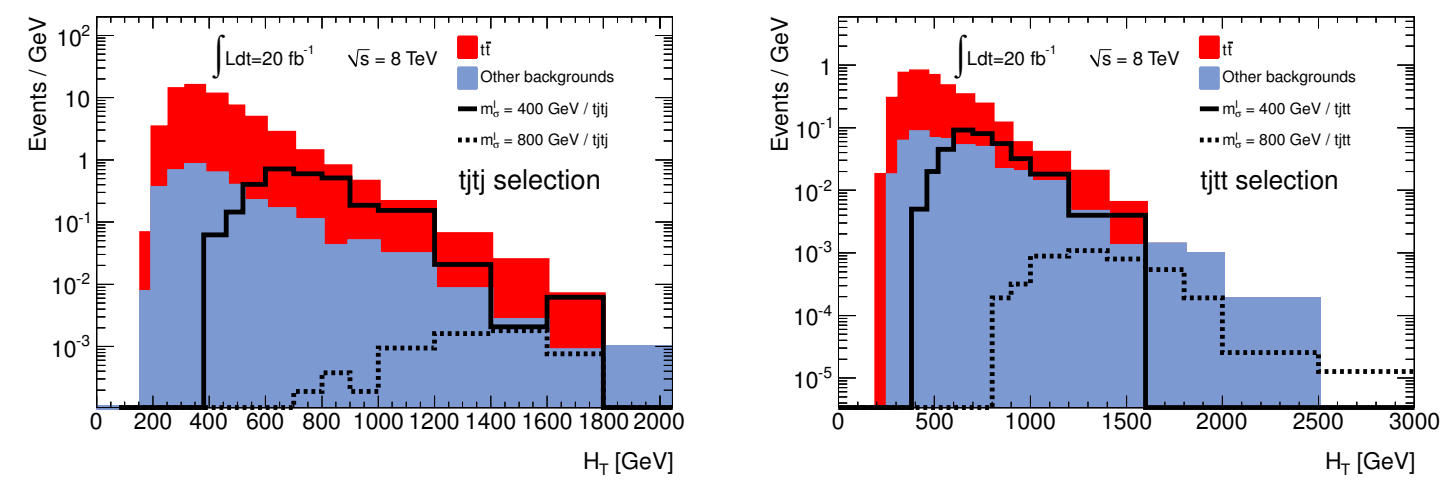

Figure 4. Distribution of the $H_{T}$ variable defined in eq. (4.2) after applying the selection strategy associated with the multilepton analysis presented in the text for the tjtj (left) and tjtt (right) topologies. We distinguish the dominant source of background associated with the production of $t \bar{t}$ pairs together with jets (red) from the other contributions (blue). We superimpose the corresponding curves for two signal scenarios of class I with respective sgluon masses of $400 \mathrm{GeV}$ (plain black curve) and $800 \mathrm{GeV}$ (dashed black curve).

to the associated production of a top-antitop pair with one or two additional gauge bosons for the $t t t t$ signature.

In our simulation setup, the multijet background, jets faking leptons and charge misidentification have not been accounted for. However, on the basis of the analysis of ref. [65] where same sign dilepton events are investigated after selection criteria similar to those applied in this paper, these sources of background have been found to contribute in a significant way as the associated number of surviving events after applying all the selections is ten times larger. We therefore adopt a conservative approach and derive, in section 4.3, two limits on sgluon-induced new physics. The first one is extracted after omitting the non-simulated backgrounds and the second one is obtained after multiplying the number of background events by a factor of ten.

We then consider the $H_{T}$ variable defined by

$$
H_{T}=\sum_{\text {jets, leptons, missing energy }}\left\|\vec{p}_{T}\right\|,
$$

since signal events are expected to contain more jets and leptons than background events. Omitting the $t t t$ channel as its statistical significance is very poor (see table 4 for illustrative benchmark scenarios), we present $H_{T}$ distributions for the $t j t j$ and $t j t t$ topologies on the left and right panels of figure 4, respectively. On both panels, we show curves associated with signal scenarios of class I where the sgluon mass is set to $400 \mathrm{GeV}$ and $800 \mathrm{GeV}$. The distributions present a steep rise once the production threshold is reached, followed by a large peak centered around twice the sgluon mass. We then compare these shapes to the corresponding background distributions, after factorizing out the dominant $t \bar{t}$ contribution (in red) from the rest of the background events (in blue). This suggests to probe the LHC sensitivity to the presence of sgluon fields coupling dominantly to top quarks by means of a 
careful investigation of the shape of the entire $H_{T}$ distributions, keeping all events, rather than requiring (unefficient) selection on this variable. Limits at the $95 \%$ confidence level are extracted in this way in section 4.3 .

\subsection{Event selection for a single lepton plus jets signature}

Final states containing exactly one lepton are expected to be copiously produced from sgluon pair production and decay at the LHC as they correspond to a fraction of events equal to $36 \%$ and $\sim 41 \%$ in the context of the $t j t j$ and $t j t t / t t t t$ topologies, respectively. We preselect events by requiring exactly one single lepton with a transverse momentum $p_{T}^{\ell}>25 \mathrm{GeV}$. Consequently, the Standard Model background is expected to be dominated at $92 \%$ by events associated with the production of a $W$-boson in association with jets. The expected number of sgluon events ranges from 34.9 (24.6) to 10700 (48.4) for a representative scenario with a sgluon mass of $400 \mathrm{GeV}(800 \mathrm{GeV})$, as shown in table 5 .

The multijet background has not been taken into account up to now. However, datadriven methods used to estimate its shape and normalization tend to show that it may have to be considered in our analysis [66]. In order to realistically and reliably reject this background, we impose, inspired by the experimental analysis of ref. [66], the missing transverse energy of the events to be larger than $\mathbb{E}_{T}>40 \mathrm{GeV}$ and the reconstructed $W$-boson transverse mass, defined as

$$
M_{T}^{W}=\sqrt{2 p_{T}^{\ell} \mathbb{E}_{T}\left[1-\cos \Delta \phi_{\ell, \mathbb{E}_{T}}\right]}
$$

to be larger than $25 \mathrm{GeV}$. In this equation, the quantity $\Delta \phi_{\ell, \mathbb{E}_{T}}$ stands for the angular distance, in the azimutal direction with respect to the beam, between the lepton and the missing energy.

As for the multilepton analysis, a higher multiplicity of hard jets is expected in signal events. They indeed arise from the hadronization of final state quarks, in contrast to the $W$ boson plus jets background where jets originate predominantly from initial-state radiation (see figure 5). Events are therefore selected with the requirement that they contain at least six, seven and eight jets with a transverse-momentum $p_{T}^{j}>25 \mathrm{GeV}$ for the $t j t j, t j t t$ and $t t t t$ topologies, respectively. Moreover, sgluon events are expected to include a higher number of heavy-flavored jets. The minimal number of $b$-tagged jets is therefore required to be larger that one and two for the $t j t j$ and $t j t t / t t t t$ search channels, respectively. At this stage, the expected Standard Model background is composed mainly of $t \bar{t}$ events, where the top-antitop pair is possibly produced in association with one or several gauge bosons.

The number of events surviving to each of the selection criteria is presented in table 5 for two representative signal scenarios with $m_{\sigma}=400 \mathrm{GeV}$ and $800 \mathrm{GeV}$ as well as for the background. After all selections, we expect a number of signal events ranging from 7.28 (8.96) to 2940 (20.4) for a sgluon mass of 400 (800) GeV according to the search channel under consideration. In contrast, the Standard Model predicts a background of 64070 , 9330 and 2658 events for the $t j t j, t j t t$ and $t t t t$ topologies.

Due to the larger activity expected in signal events compared to background events, we consider, as in section 4.1, the $H_{T}$ variable as a discriminant between signal and back- 


\begin{tabular}{|c|ccc|}
\hline Selections & \multicolumn{3}{|c|}{$t j t j$ channel } \\
& $m_{\sigma}^{I}=400 \mathrm{GeV}$ & $m_{\sigma}^{I}=800 \mathrm{GeV}$ & Backgrounds \\
\hline$N_{\ell}=1$ with $p_{T}^{\ell} \geq 25 \mathrm{GeV}$ & $(1.06 \pm 0.01) \cdot 10^{4}$ & $45.7 \pm 0.9$ & $(2.376 \pm 0.003) \cdot 10^{8}$ \\
$\mathbb{E}_{T} \geq 40 \mathrm{GeV}$ & $(7.65 \pm 0.06) \cdot 10^{3}$ & $37.9 \pm 0.8$ & $(6.836 \pm 0.002) \cdot 10^{7}$ \\
$M_{T}^{W} \geq 25 \mathrm{GeV}$ & $(6.43 \pm 0.05) \cdot 10^{3}$ & $30.7 \pm 0.7$ & $(6.722 \pm 0.002) \cdot 10^{7}$ \\
$N_{j} \geq 6$ with $p_{T}^{j} \geq 25 \mathrm{GeV}$ & $(3.88 \pm 0.04) \cdot 10^{3}$ & $24.9 \pm 0.7$ & $(8.634 \pm 0.024) \cdot 10^{4}$ \\
$N_{b} \geq 1$ & $(2.91 \pm 0.04) \cdot 10^{3}$ & $19.3 \pm 0.6$ & $(6.407 \pm 0.014) \cdot 10^{4}$ \\
\hline
\end{tabular}

\begin{tabular}{|c|ccc|}
\hline Selections & \multicolumn{3}{|c|}{$t j t t$ channel } \\
& $m_{\sigma}^{I}=400 \mathrm{GeV}$ & $m_{\sigma}^{I}=800 \mathrm{GeV}$ & Backgrounds \\
\hline$N_{\ell}=1$ with $p_{T}^{\ell} \geq 25 \mathrm{GeV}$ & $(1.21 \pm 0.22) \cdot 10^{3}$ & $21.3 \pm 0.4$ & $(2.376 \pm 0.001) \cdot 10^{8}$ \\
$\mathscr{E}_{T} \geq 40 \mathrm{GeV}$ & $(8.81 \pm 0.19) \cdot 10^{2}$ & $18.1 \pm 0.3$ & $(6.836 \pm 0.002) \cdot 10^{7}$ \\
$M_{T}^{W} \geq 25 \mathrm{GeV}$ & $(7.66 \pm 0.18) \cdot 10^{2}$ & $15.4 \pm 0.3$ & $(6.722 \pm 0.002) \cdot 10^{7}$ \\
$N_{j} \geq 7$ with $p_{T}^{j} \geq 25 \mathrm{GeV}$ & $(4.05 \pm 0.13) \cdot 10^{2}$ & $11.08 \pm 0.3$ & $(2.613 \pm 0.012) \cdot 10^{4}$ \\
$N_{b} \geq 2$ & $(1.99 \pm 0.09) \cdot 10^{2}$ & $5.99 \pm 0.2$ & $(9.330 \pm 0.050) \cdot 10^{3}$ \\
\hline
\end{tabular}

\begin{tabular}{|c|ccc|}
\hline Selections & \multicolumn{3}{|c|}{ tttt channel } \\
& $m_{\sigma}^{I}=400 \mathrm{GeV}$ & $m_{\sigma}^{I I}=800 \mathrm{GeV}$ & Backgrounds \\
\hline$N_{\ell}=1$ with $p_{T}^{\ell} \geq 25 \mathrm{GeV}$ & $34.6 \pm 0.6$ & $23.2 \pm 0.4$ & $(2.376 \pm 0.001) \cdot 10^{8}$ \\
$\mathbb{E}_{T} \geq 40 \mathrm{GeV}$ & $27.3 \pm 0.5$ & $20.2 \pm 0.4$ & $(6.836 \pm 0.002) \cdot 10^{7}$ \\
$M_{T}^{W} \geq 25 \mathrm{GeV}$ & $23.6 \pm 0.5$ & $17.1 \pm 0.3$ & $(6.722 \pm 0.002) \cdot 10^{7}$ \\
$N_{j} \geq 8$ with $p_{T}^{j} \geq 25 \mathrm{GeV}$ & $10.8 \pm 0.3$ & $12.3 \pm 0.3$ & $(7.020 \pm 0.060) \cdot 10^{3}$ \\
$N_{b} \geq 2$ & $7.21 \pm 0.27$ & $8.47 \pm 0.23$ & $(2.658 \pm 0.026) \cdot 10^{3}$ \\
\hline
\end{tabular}

Table 5. Same as table 4 but in the context of a single lepton final state. For a detailed description of each of the selection criteria, we refer to the text.

ground. Following the definition of eq. (4.2), we present, in figure 6, the $H_{T}$ distributions after following the $t j t t$ (left panel) and $t t t t$ (right panel) single lepton selection strategy introduced above. We show background distributions after distinguishing events associated with top-antitop production in association with jets (red) from the rest of the background (blue). The resulting behavior underlines a steep rise once the top-antitop production threshold is reached, both in the $t j t t$ and $t t t t$ channels, followed by a peak around $H_{T} \sim 500 \mathrm{GeV}$ and a smooth fall with increasing energy. In comparison, signal distributions are associated with a clear peaky behavior centered around an $H_{T}$ value depending on the sgluon mass $\left(\sim 1.5 m_{\sigma}\right)$ and the tail of the distributions does not extend to a very large hadronic energy, in contrast to the Standard Model background expectation. Instead of requiring a selection criterion based on the $H_{T}$ variable, we then employ the shape of the associated distributions as inputs to extract the LHC sgluon mass reach in section 4 . 


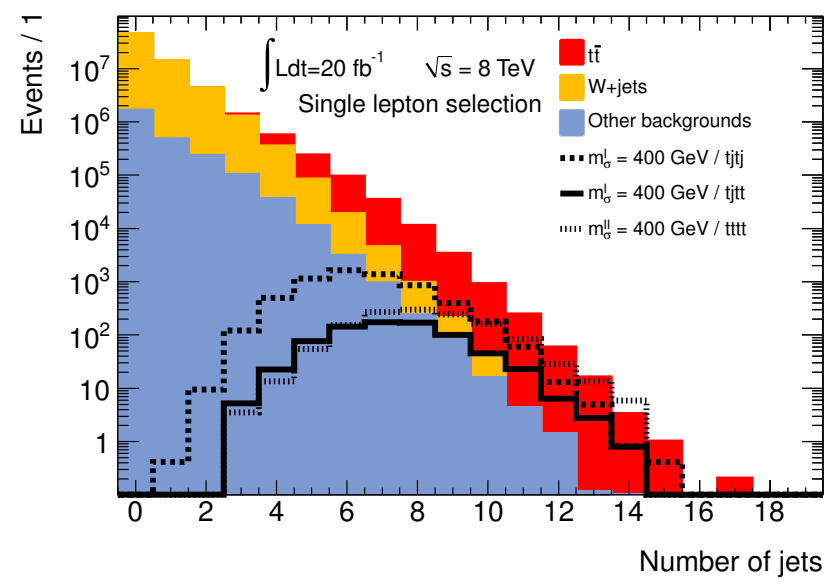

Figure 5. Jet multiplicity distribution after selecting events with exactly one lepton, missing transverse energy $\mathbb{E}_{T}>40 \mathrm{GeV}$ and a $W$-boson transverse mass $M_{T}^{W}>25 \mathrm{GeV}$. We distinguish the $t \bar{t}$ (red) and $W$-boson plus jets (orange) contributions from the rest of the background (blue) and present signal distributions for a sgluon scenarios of class I with $m_{\sigma}=400 \mathrm{GeV}$ in the $t j t j$ (plain), tjtt (strong dashed) and tttt (light dashed) channels.
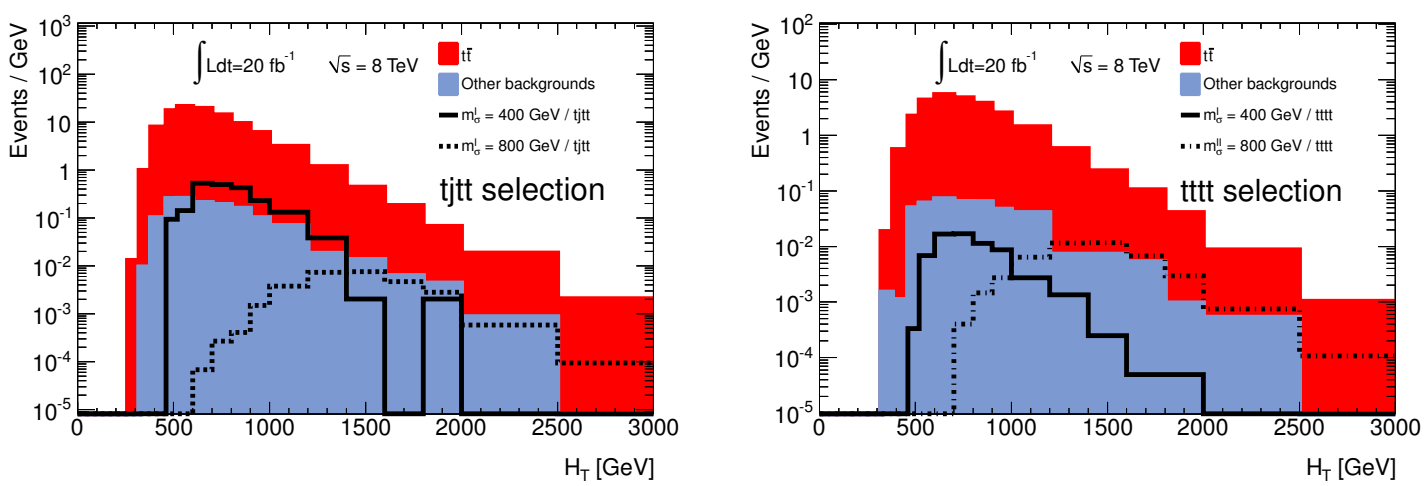

Figure 6. Distribution of the $H_{T}$ variable defined in eq. (4.2) after the selection strategy associated to a single lepton analysis as presented in the text. We distinguish the background events associated to the production of $t \bar{t}$ pairs together with jets (red) from the other contributions (blue). For the $t j t t$ channel (left panel), we superimpose the corresponding curves for two signal scenarios of class I with respective sgluon masses of $400 \mathrm{GeV}$ (plain black curve) and $800 \mathrm{GeV}$ (dashed black curve). In the case of the $t t t$ search-channel (right panel), we rather consider a scenario of class I with a sgluon mass of $400 \mathrm{GeV}$ (plain black curve) and a scenario of class II with a sgluon mass of $800 \mathrm{GeV}$ (dashed black curve).

In the case of a $t j t j$ signature with exactly one final state lepton, the reconstruction of the sgluon mass is possible if the missing energy is assumed to originate only from the neutrino issued from the $W$-boson decay. Assigning the labeling of the six jets according 
to the process

$$
p p \rightarrow \sigma \sigma \rightarrow\left(t j_{5}\right)\left(t j_{6}\right) \rightarrow\left(j_{1} j_{2} j_{3} j_{5}\right)\left(j_{4} \ell \nu j_{6}\right),
$$

all possible permutations of the six jets are performed and the one minimizing a $\chi^{2}$-variable defined by

$$
\begin{aligned}
\chi^{2}= & {\left[\frac{m_{j_{1} j_{2}}-m_{W}^{(r)}}{\sigma_{W}^{(r)}}\right]^{2}+\left[\frac{\left(m_{j_{1} j_{2} j_{3}}-m_{j_{1} j_{2}}\right)-m_{t W}^{(r)}}{\sigma_{t W}^{(r)}}\right]^{2}+\left[\frac{m_{\ell \nu j_{4}}-m_{t \ell}^{(r)}}{\sigma_{t \ell}^{(r)}}\right]^{2}+} \\
& +\left[\frac{\left(m_{\ell \nu j_{4}, j_{6}}-m_{\ell \nu j_{4}}\right)-\left(m_{j_{1} j_{2} j_{3}, j_{5}}-m_{j_{1} j_{2} j_{3}}\right)}{\sigma_{\sigma t}^{(r)}\left[\left(m_{\ell \nu j_{4}, j_{6}}-m_{\ell \nu j_{4}}\right)+\left(m_{j_{1} j_{2} j_{3}, j_{5}}-m_{j_{1} j_{2} j_{3}}\right)\right]}\right]^{2}
\end{aligned}
$$

is retained as the true configuration of a given event. The analytical expression of this $\chi^{2}$-variable exactly mimics eq. (4.4). The three jets $j_{1}, j_{2}$ and $j_{3}$ are the ones issued from the hadronically decaying top quark, $j_{3}$ being a $b$-jet, ${ }^{2}$ and this information is encompassed into the first two terms of the $\chi^{2}$ variable. The invariant mass of the two light jets $j_{1}$ and $j_{2}$, denoted by $m_{j_{1} j_{2}}$, is asked to be compatible with the $W$-boson mass and the three-jet invariant mass $m_{j_{1} j_{2} j_{3}}$ is required to be compatible with the top mass. However, in order not to introduce a correlation between the first two terms of the $\chi^{2}$ variable, we subtract from the reconstructed top mass $m_{j_{1} j_{2} j_{3}}$ the reconstructed dijet invariant-mass $m_{j_{1} j_{2}}$. The values of the fit parameters are taken as $m_{W}^{(r)}=80.7 \mathrm{GeV}, \sigma_{W}^{(r)}=8.9 \mathrm{GeV}, m_{t W}^{(r)}=90.8 \mathrm{GeV}$ and $\sigma_{t W}^{(r)}=10.5 \mathrm{GeV}$. These numerical values have been extracted from a fit based on the Monte Carlo truth, which ensures that each reconstructed object is correctly assigned according to the configuration of eq. (4.4). In our simulation setup, it must be noted that the large values of $\mathcal{O}(10 \%)$ for the widths are mainly related to detector resolution.

In the third term of eq. (4.5), we focus on the leptonically decaying top quark and ask the invariant mass $m_{\ell \nu j_{4}}$ to be compatible with the top mass. Since the neutrino fourmomentum is entirely reconstructed from the assumption that both the missing energy and the charged lepton are issued from the decay of a $W$-boson, there is no need to add an extra term in the definition of the $\chi^{2}$, the information being already implicitly included in the $m_{\ell \nu j_{4}}$ term. From the Monte Carlo truth, we have calculated a reconstructed mass equal to $m_{t l}^{(r)}=167.8 \mathrm{GeV}$, together with a width of $\sigma_{t l}^{(r)}=19.1 \mathrm{GeV}$.

Finally, the definition of the $\chi^{2}$ variable of eq. (4.5) exploits the fact that both the $t j_{5}$ and $t j_{6}$ pairs are issued from the decay of a sgluon field. The corresponding invariant masses $m_{j_{1} j_{2} j_{3}, j_{5}}$ and $m_{\ell \nu j_{4}, j_{6}}$ must hence be self-compatible, up to the detector resolution. This is translated in the last term of eq. (4.5), after subtracting the respective reconstructed top masses to avoid possible correlations among the different terms of the $\chi^{2}$. From the Monte Carlo truth, we fix the related width to $\sigma_{\sigma t}^{(r)}=0.098 \mathrm{GeV}$.

Performing an event selection as described in the upper panel of table 5 and minimizing the $\chi^{2}$ variable of eq. (4.5), each event is reconstructed according to the pattern given in eq. (4.4). This allows us to extract the sgluon mass $m_{\sigma}^{(r)}$. The resulting distributions are presented on figure 7 for both the background and four signal scenarios of class I with a sgluon mass parameter $m_{\sigma}$ taken equal to $200 \mathrm{GeV}, 400 \mathrm{GeV}, 600 \mathrm{GeV}$ and $800 \mathrm{GeV}$, respectively. As expected, the background distribution, where we again distinguish the

\footnotetext{
${ }^{2}$ We are however not making use of jet flavor information in our kinematical fit.
} 


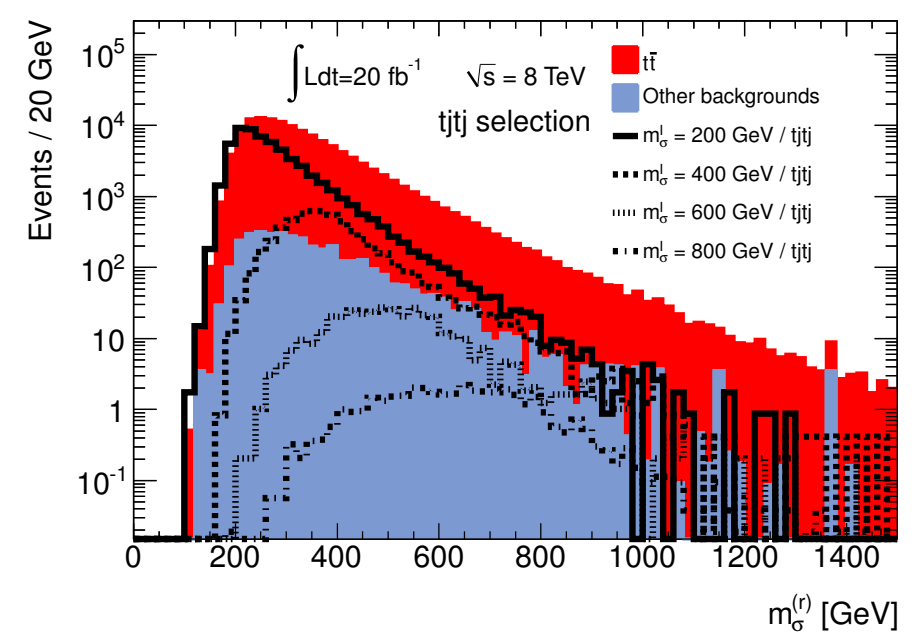

Figure 7. Reconstructed sgluon mass $m_{\sigma}^{(r)}$ for both the background and several signal scenarios in the context of the $t j t j$ topology. Event selection is performed as in the upper panel of table 5 and we reconstruct each event according to the pattern given in eq. (4.4) by means of the minimization of the $\chi^{2}$ variable of eq. (4.5). Concerning the background distribution, we distinguish the dominant top-antitop contribution (red) from the rest of the background (blue) and superimpose signal curves for four scenarios of class I, with respective sgluon mass of $200 \mathrm{GeV}$ (plain), $400 \mathrm{GeV}$ (strong dotted), $600 \mathrm{GeV}$ (light dotted) and $800 \mathrm{GeV}$ (dash-dotted).

top-antitop pair contributions (in red) from the other sources of background (in blue), presents a rising behavior once the production threshold of a top-antitop pair is reached, quickly followed by a slow fall which extends to rather large values of the reconstructed sgluon mass $m_{\sigma}^{(r)}$. In contrast, the signal distributions all show a peak. For light sgluons $\left(m_{\sigma}=200 \mathrm{GeV}\right.$ or $\left.400 \mathrm{GeV}\right)$, this peak is clearly centered around the true sgluon mass. In the case of heavier sgluons $\left(m_{\sigma}=600 \mathrm{GeV}\right.$ and $\left.800 \mathrm{GeV}\right)$, the detector resolution renders the peak very wide and centered around a value equal to about $70 \%-80 \%$ of the real sgluon mass. We then use, in the next section, the reconstructed mass $m_{\sigma}^{(r)}$ to extract limits on the sgluon mass reachable at the LHC.

\subsection{LHC sensitivity to a sgluon field dominantly coupling to top quarks}

For each of the final states considered in this paper, we combine the number of expected signal and background events, including their corresponding statistical uncertainties, to calculate upper limits on the signal cross section at the $95 \%$ confidence level. To this aim, we use the CLs technique [67] as implemented in the MCLimit software [68]. We employ the $H_{T}$ variable defined in eq. (4.2) to discriminate signal from background in the multilepton (for the $t j t j, t j t t$ and $t t t t$ topologies) and single lepton (for the $t j t t$ and $t t t t$ topologies) analyses while the reconstructed sgluon mass is chosen in the case of a single lepton analysis applied to a $t j t j$ final state. ${ }^{3}$

\footnotetext{
${ }^{3}$ We have checked that considering the reconstructed mass instead of the $H_{T}$ variable allows us to improve the LHC sensitivity by about $15 \%-20 \%$ in the low mass region. This choice is however irrelevant for the higher sgluon mass region.
} 


\begin{tabular}{|c|c|c|c|}
\hline & Single lepton analysis & Multilepton analysis & $\begin{array}{c}\text { Multilepton analysis } \\
\text { (background } \times 10)\end{array}$ \\
\hline$t j t j$ & $590_{-30}^{+40} \mathrm{GeV}$ & $570_{-50}^{+30} \mathrm{GeV}$ & $4400_{-15}^{+40} \mathrm{GeV}$ \\
$t j t t$ & $480{ }_{-80}^{+70} \mathrm{GeV}$ & $520_{-90}^{+35} \mathrm{GeV}$ & - \\
$t t t t$ (Scenario I) & - & - & - \\
$t t t t$ (Scenario II) & $640_{-30}^{+40} \mathrm{GeV}$ & $650_{-40}^{+30} \mathrm{GeV}$ & $520_{-110}^{+50} \mathrm{GeV}$ \\
\hline
\end{tabular}

Table 6. Expected sensitivity of the LHC collider, running at a center-of-mass of $8 \mathrm{TeV}$ and for an integrated luminosity of $20 \mathrm{fb}^{-1}$, to sgluons. The results are given, together with the associated $1 \sigma$ statistical uncertainties, in terms of upper bounds on the sgluon mass to be reached, at the 95\% confidence level, for different types of analyses and scenarios. For scenarios of class I, the expectations for the $t j t j$ channel are given on the first line of the table, those related to the $t j t t$ channel on its second line and those associated with the $t t t t$ channel on its third line. Scenarios of class II are only investigated in the context of the ttt search-channel and results are shown on the fourth line of the table. Finally, we distinguish results obtained by employing a single lepton analysis (second column), a multilepton analysis after neglecting the QCD background (third column) and a multilepton analysis after multiplying the simulated background by a factor of ten (fourth column).

The results are presented in figure 8 for the $t j t j$ channel (upper panel), $t j t t$ channel (middle panel) and tttt channel (lower panel) as dashed and dot-dashed curves in the context of the multilepton and single lepton analysis, respectively. In addition, the effects of the non-simulated sources of background mentioned in section 4.1 are presented as dotted curves. On these figures, we also show the theoretical cross sections related to sgluon-induced production of multitop final states as a function of the sgluon mass. In addition to the central NLO curves derived from table 2, we include a $30 \%$ uncertainty band corresponding to the typical order of magnitude of the variations of the results with respect to different choices for the factorization and renormalization scales [44]. The curves associated with scenarios of class I are shown in dark gray, while those related to scenarios of class II are given in light gray.

Sgluon masses excluded at the $95 \%$ confidence level are also indicated in table 6 , for each final state and for each scenario considered in this paper. The quoted uncertainties correspond to an estimation of the cross section limits at the $1 \sigma$ level, where $\sigma$ stands for the statistical uncertainty.

In the multilepton analysis, sgluon masses lower than 570 and $520 \mathrm{GeV}$ can be excluded in the $t j t j$ and $t j t t$ topologies, respectively, for scenarios of class I. Equivalently, the ATLAS experiment is sensitive to sgluon-induced multitop production cross sections of $\mathcal{O}(100) \mathrm{fb}$ for both the $t j t j$ and $t j t t$ signatures at $\sqrt{s}=8 \mathrm{TeV}$. Inspecting the second column of table 6 , the limits obtained for the $t j t j$ search-channel barely vary (by less than 10\%) when accounting for $1 \sigma$ statistical uncertainties. In contrast, the bounds extracted from the analysis of the $t j t t$ topology are found to be more sensitive to statistics as they can vary by about $17 \%$ with respect to (un)lucky fluctuations. This feature can be understood from the behavior 

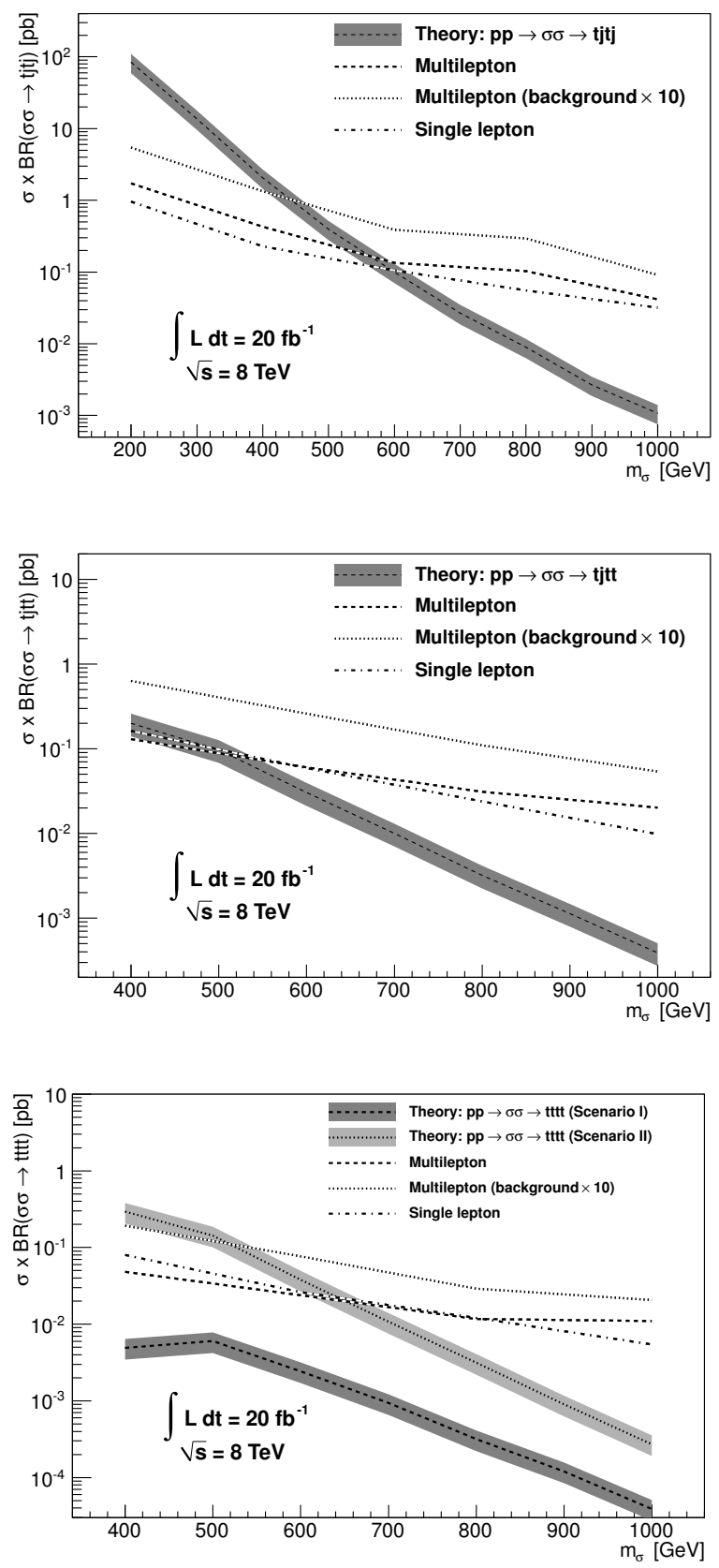

Figure 8. The $95 \%$ confidence level expected signal cross sections as a function of the sgluon mass, for an integrated luminosity of $20 \mathrm{fb}^{-1}$ and $\sqrt{s}=8 \mathrm{TeV}$. The bands around the theoretical curves correspond to an uncertainty of $30 \%$, and predictions for scenarios of class I (II) are presented as light (dark) gray bands. The expected limits in the $t j t j$ (upper), $t j t t$ (middle) and $t t t t$ channels (lower) are given, for the single lepton (multilepton) analysis, as dot-dashed (dashed) curves. In the multilepton case, we also show the results obtained after enhancing the background by a factor of ten (dotted curves). 
of the theoretical cross sections in the $500-600 \mathrm{GeV}$ sgluon mass range. While the $t j t j$ cross section decreases with the sgluon mass, the $t j t t$ cross section is rather flat. The multilepton analysis is however not sensitive to sgluon-induced production of four top quarks for class I scenarios, at $\sqrt{s}=8 \mathrm{TeV}$ and for an integrated luminosity of $20 \mathrm{fb}^{-1}$. This results from the too low branching ratio of the sgluon decay into a top-antitop pair (see table 2). In class II scenarios, this branching ratio is 2.5-7.6 times more important, so that sgluon masses lower than $650 \mathrm{GeV}$, or cross section of $\mathcal{O}(10) \mathrm{fb}$, can be excluded at the $95 \%$ confidence level.

When taking into account non-simulated backgrounds, the total number of expected background events is conservatively multiplied by ten. Consequently, the $t j t t$ analysis is not sensitive to sgluon-pair production anymore, while the masses excluded when analyzing $t j t j$ and $t t t$ (for scenarios of class II) final states decrease from 570 to $440 \mathrm{GeV}$ and 650 to $520 \mathrm{GeV}$, respectively.

We now turn to single lepton analyses and show that the reconstructed sgluon mass from a $t j t j$ topology can be used to exclude, at the $95 \%$ confidence level, sgluon as heavy as $590 \mathrm{GeV}$ (dot-dashed curve). Moreover, these bounds are found not to drastically vary when including $1 \sigma$ statistical uncertainties. Concerning the $t j t t$ and $t t t t$ topologies, the $H_{T}$ variable is considered as a discriminant and sgluon masses up to $480 \mathrm{GeV}$ and $640 \mathrm{GeV}$ can be reached. In the first case, statistical fluctuations can lead to different expectations by about $\pm 15 \%$ in the $t j t t$ case while in the $t t t t$ case, the results are found only to slightly change by about $5 \%$.

\section{Conclusion}

Many new physics theories predict the existence of a scalar field, commonly dubbed sgluon, lying in the adjoint representation of the QCD gauge group. To investigate the sensitivity of the LHC to this particle in the case it couples dominantly to the top quark, as motivated by hybrid $N=1 / N=2$ or $R$-symmetric supersymmetric theories, an effective field theory has been built. This theory consists of a minimal extension of the Standard Model including a single real sgluon field, together with a set of interactions leading to its production and decay at the LHC. The model has been implemented in the FEYNRules package so that a UFO model for MADGRAPH 5 has been extracted.

Final states containing two, three, or four top quarks produced in association with jets have been investigated. To optimize the sensitivity of the search for sgluon-pair production predominantly decaying to top quarks, two signatures (multilepton and single lepton) have been considered in three topologies $(t j t j, t j t t, t t t t)$. We have shown that sgluons of about $500-700 \mathrm{GeV}$ can be reached at the LHC collider running at a center-of-mass energy of $8 \mathrm{TeV}$ and for an integrated luminosity of $20 \mathrm{fb}^{-1}$. Equivalently, this mass constraint can be translated into a bound on the cross section associated with the production of two, three, or four top quarks issued from the decays of a pair of sgluons. Contributions higher than 10-100 fb can be excluded for most scenarios, at the $95 \%$ confidence level. While a search strategy based on a single lepton selection ensures, with appropriate selection criteria, the multijet background to be under good control, non-simulated contributions to the background such as jets faking leptons and charge misidentification can reduce the sensitivity of the multilepton signature by several hundreds of $\mathrm{GeV}$. 
Our work therefore motivates a future extension of the two performed analyses in the context of a full detector simulation of the LHC experiments.

\section{Acknowledgments}

The authors are grateful to D. Zerwas for enlightening discussions at the beginning of this project. Moreover, we thank the entire MADGolem team, and more in particular D. Gonçalves Netto, D. López-Val and K. Mawatari, for helpful and interesting discussions as well as for providing us next-to-leading order $K$-factors. This work has been supported by the Theory-LHC-France initiative of the CNRS/IN2P3 and a Ph.D. fellowship of the French ministry for education and research. BF acknowledges partial support from the French ANR 12 JS05 00201 BATS@LHC.

Open Access. This article is distributed under the terms of the Creative Commons Attribution License which permits any use, distribution and reproduction in any medium, provided the original author(s) and source are credited.

\section{References}

[1] ATLAS collaboration, Observation of a new particle in the search for the Standard Model Higgs boson with the ATLAS detector at the LHC, Phys. Lett. B 716 (2012) 1 [arXiv: 1207.7214] [INSPIRE].

[2] CMS collaboration, Observation of a new boson at a mass of $125 \mathrm{GeV}$ with the CMS experiment at the LHC, Phys. Lett. B 716 (2012) 30 [arXiv:1207.7235] [INSPIRE].

[3] H.P. Nilles, Supersymmetry, supergravity and particle physics, Phys. Rept. 110 (1984) 1 [INSPIRE].

[4] H.E. Haber and G.L. Kane, The search for supersymmetry: probing physics beyond the Standard Model, Phys. Rept. 117 (1985) 75 [InSPIRE].

[5] ATLAS and CMS collaborations, Supersymmetry searches with ATLAS and CMS, arXiv: 1205.4053 [INSPIRE].

[6] ATLAS supersymmetry (SUSY) searches webpage, https://twiki.cern.ch/twiki/bin/view/AtlasPublic/SupersymmetryPublicResults.

[7] CMS supersymmetry physics results webpage, https://twiki.cern.ch/twiki/bin/view/CMSPublic/PhysicsResultsSUS.

[8] P. Fayet, Fermi-Bose hypersymmetry, Nucl. Phys. B 113 (1976) 135 [InSPIRE].

[9] L. Álvarez-Gaumé and S. Hassan, Introduction to $S$ duality in $N=2$ supersymmetric gauge theories: a pedagogical review of the work of Seiberg and Witten, Fortsch. Phys. 45 (1997) 159 [hep-th/9701069] [INSPIRE].

[10] T. Plehn and T.M.P. Tait, Seeking sgluons, J. Phys. G 36 (2009) 075001 [arXiv:0810.3919] [INSPIRE].

[11] S. Choi, M. Drees, A. Freitas and P. Zerwas, Testing the Majorana nature of gluinos and neutralinos, Phys. Rev. D 78 (2008) 095007 [arXiv:0808.2410] [INSPIRE].

[12] S. Choi et al., Color-octet scalars of $N=2$ supersymmetry at the LHC, Phys. Lett. B 672 (2009) 246 [arXiv:0812.3586] [INSPIRE]. 
[13] S. Choi et al., Color-octet scalars at the LHC, Acta Phys. Polon. B 40 (2009) 1947 [arXiv:0902.4706] [INSPIRE].

[14] S. Choi et al., Dirac neutralinos and electroweak scalar bosons of $N=1 / N=2$ hybrid supersymmetry at colliders, JHEP 08 (2010) 025 [arXiv: 1005.0818] [INSPIRE].

[15] S. Choi, D. Choudhury, A. Freitas, J. Kalinowski and P. Zerwas, The extended Higgs system in R-symmetric supersymmetry theories, Phys. Lett. B 697 (2011) 215 [Erratum ibid. B 698 (2011) 457] [arXiv: 1012.2688] [INSPIRE].

[16] S. Schumann, A. Renaud and D. Zerwas, Hadronically decaying color-adjoint scalars at the LHC, JHEP 09 (2011) 074 [arXiv:1108.2957] [InSPIRE].

[17] W. Kotlarski and J. Kalinowski, Scalar gluons at the LHC, Acta Phys. Polon. B 42 (2011) 2485 [inSPIRE].

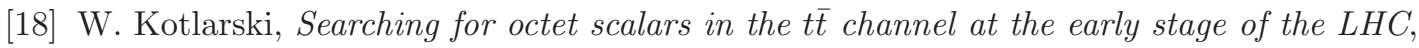
Acta Phys. Polon. B 42 (2011) 1457 [InSPIRE].

[19] A. Salam and J. Strathdee, Supersymmetry and fermion number conservation, Nucl. Phys. B 87 (1975) 85 [InSPIRE].

[20] P. Fayet, Supergauge invariant extension of the Higgs mechanism and a model for the electron and its neutrino, Nucl. Phys. B 90 (1975) 104 [INSPIRE].

[21] G.D. Kribs, E. Poppitz and N. Weiner, Flavor in supersymmetry with an extended R-symmetry, Phys. Rev. D 78 (2008) 055010 [arXiv:0712.2039] [INSPIRE].

[22] C. Kilic, T. Okui and R. Sundrum, Colored resonances at the Tevatron: phenomenology and discovery potential in multijets, JHEP 07 (2008) 038 [arXiv:0802.2568] [INSPIRE].

[23] C. Kilic, S. Schumann and M. Son, Searching for multijet resonances at the LHC, JHEP 04 (2009) 128 [arXiv:0810.5542] [INSPIRE].

[24] C. Kilic, T. Okui and R. Sundrum, Vectorlike confinement at the LHC, JHEP 02 (2010) 018 [arXiv:0906.0577] [INSPIRE].

[25] C. Kilic and T. Okui, The LHC phenomenology of vectorlike confinement, JHEP 04 (2010) 128 [arXiv: 1001.4526] [INSPIRE].

[26] D.A. Dicus, C. Kao, S. Nandi and J. Sayre, Discovering colorons at the early stage LHC, Phys. Rev. D 83 (2011) 091702 [arXiv: 1012.5694] [InSPIRE].

[27] J. Sayre, D.A. Dicus, C. Kao and S. Nandi, Searching for colorons at the Large Hadron Collider, Phys. Rev. D 84 (2011) 015011 [arXiv:1105.3219] [INSPIRE].

[28] G. Burdman, B.A. Dobrescu and E. Ponton, Resonances from two universal extra dimensions, Phys. Rev. D 74 (2006) 075008 [hep-ph/0601186] [INSPIRE].

[29] LHC New Physics Working Group collaboration, D. Alves et al., Simplified models for LHC new physics searches, J. Phys. G 39 (2012) 105005 [arXiv:1105.2838] [INSPIRE].

[30] G. Brooijmans et al., Les Houches 2011: physics at TeV colliders new physics working group report, arXiv: 1203.1488 [INSPIRE].

[31] ATLAS collaboration, Search for new physics in the dijet mass distribution using $1 \mathrm{fb}^{-1}$ of pp collision data at $\sqrt{s}=7 \mathrm{TeV}$ collected by the ATLAS detector,

Phys. Lett. B 708 (2012) 37 [arXiv:1108.6311] [INSPIRE]. 
[32] ATLAS collaboration, Search for massive colored scalars in four-jet final states in $\sqrt{s}=7$ TeV proton-proton collisions with the ATLAS detector,

Eur. Phys. J. C 71 (2011) 1828 [arXiv:1110.2693] [INSPIRE].

[33] ATLAS collaboration, Search for massive coloured scalars with the ATLAS detector in four-jet final states using $4.6 \mathrm{fb}^{-1}$ of $\sqrt{s}=7 \mathrm{TeV}$ proton-proton collision data, ATLAS-CONF-2012-110, CERN, Geneva Switzerland (2012).

[34] CMS collaboration, Search for narrow resonances using the dijet mass spectrum in pp collisions at $\sqrt{s}=8 \mathrm{TeV}$, CMS-PAS-EXO-12-016, CERN, Geneva Switzerland (2012).

[35] N.D. Christensen et al., A comprehensive approach to new physics simulations, Eur. Phys. J. C 71 (2011) 1541 [arXiv:0906.2474] [INSPIRE].

[36] N.D. Christensen and C. Duhr, FeynRules - Feynman rules made easy, Comput. Phys. Commun. 180 (2009) 1614 [arXiv:0806.4194] [INSPIRE].

[37] N.D. Christensen, C. Duhr, B. Fuks, J. Reuter and C. Speckner, Introducing an interface between WHIZARD and FeynRules, Eur. Phys. J. C 72 (2012) 1990 [arXiv:1010.3251] [INSPIRE].

[38] C. Duhr and B. Fuks, A superspace module for the FeynRules package, Comput. Phys. Commun. 182 (2011) 2404 [arXiv:1102.4191] [INSPIRE].

[39] B. Fuks, Beyond the minimal supersymmetric Standard Model: from theory to phenomenology, Int. J. Mod. Phys. A 27 (2012) 1230007 [arXiv:1202.4769] [InSPIRE].

[40] C. Degrande et al., UFO - the Universal FeynRules Output, Comput. Phys. Commun. 183 (2012) 1201 [arXiv:1108.2040] [INSPIRE].

[41] J. Alwall, M. Herquet, F. Maltoni, O. Mattelaer and T. Stelzer, MadGraph 5: going beyond, JHEP 06 (2011) 128 [arXiv:1106.0522] [INSPIRE].

[42] J. Pumplin et al., New generation of parton distributions with uncertainties from global QCD analysis, JHEP 07 (2002) 012 [hep-ph/0201195] [INSPIRE].

[43] T. Hahn, Generating Feynman diagrams and amplitudes with FeynArts 3, Comput. Phys. Commun. 140 (2001) 418 [hep-ph/0012260] [InSPIRE].

[44] D. Goncalves-Netto, D. Lopez-Val, K. Mawatari, T. Plehn and I. Wigmore, Sgluon pair production to next-to-leading order, Phys. Rev. D 85 (2012) 114024 [arXiv:1203.6358] [INSPIRE].

[45] S. Ovyn, X. Rouby and V. Lemaitre, DELPHES, a framework for fast simulation of a generic collider experiment, arXiv:0903.2225 [INSPIRE].

[46] M. Cacciari and G.P. Salam, Dispelling the $N^{3}$ myth for the $k_{t}$ jet-finder, Phys. Lett. B 641 (2006) 57 [hep-ph/0512210] [INSPIRE].

[47] LHC performance workshop Chamonix France 2012 webpage, https://indico.cern.ch/internalPage.py?pageId=3\&confId=164089.

[48] T. Sjöstrand, S. Mrenna and P.Z. Skands, PYTHIA 6.4 physics and manual, JHEP 05 (2006) 026 [hep-ph/0603175] [INSPIRE].

[49] M.L. Mangano, M. Moretti, F. Piccinini and M. Treccani, Matching matrix elements and shower evolution for top-quark production in hadronic collisions, JHEP 01 (2007) 013 [hep-ph/0611129] [INSPIRE]. 
[50] J. Alwall, S. de Visscher and F. Maltoni, QCD radiation in the production of heavy colored particles at the LHC, JHEP 02 (2009) 017 [arXiv: 0810.5350] [INSPIRE].

[51] N. Davidson, G. Nanava, T. Przedzinski, E. Richter-Was and Z. Was, Universal interface of TAUOLA technical and physics documentation, Comput. Phys. Commun. 183 (2012) 821 [arXiv: 1002.0543] [INSPIRE].

[52] K. Melnikov and F. Petriello, Electroweak gauge boson production at hadron colliders through O( $\left.\alpha_{s}^{2}\right)$, Phys. Rev. D 74 (2006) 114017 [hep-ph/0609070] [INSPIRE].

[53] R. Gavin, Y. Li, F. Petriello and S. Quackenbush, $W$ physics at the LHC with FEWZ 2.1, arXiv: 1201.5896 [INSPIRE].

[54] R. Gavin, Y. Li, F. Petriello and S. Quackenbush, FEWZ 2.0: a code for hadronic Z production at next-to-next-to-leading order, Comput. Phys. Commun. 182 (2011) 2388 [arXiv: 1011.3540] [INSPIRE].

[55] H.-L. Lai et al., New parton distributions for collider physics, Phys. Rev. D 82 (2010) 074024 [arXiv: 1007.2241] [InSPIRE].

[56] M. Aliev et al., HATHOR: HAdronic Top and Heavy quarks crOss section calculatoR, Comput. Phys. Commun. 182 (2011) 1034 [arXiv:1007.1327] [INSPIRE].

[57] P. Baernreuther, M. Czakon and A. Mitov, Percent level precision physics at the Tevatron: first genuine NNLO QCD corrections to $q \bar{q} \rightarrow t \bar{t}+X$, Phys. Rev. Lett. 109 (2012) 132001 [arXiv: 1204.5201] [INSPIRE].

[58] N. Kidonakis, NNLL resummation for s-channel single top quark production, Phys. Rev. D 81 (2010) 054028 [arXiv: 1001.5034] [INSPIRE].

[59] N. Kidonakis, Two-loop soft anomalous dimensions for single top quark associated production with a $W^{-}$or $H^{-}$, Phys. Rev. D 82 (2010) 054018 [arXiv: 1005.4451] [INSPIRE].

[60] N. Kidonakis, Next-to-next-to-leading-order collinear and soft gluon corrections for t-channel single top quark production, Phys. Rev. D 83 (2011) 091503 [arXiv:1103.2792] [INSPIRE].

[61] N. Kidonakis, Differential and total cross sections for top pair and single top production, arXiv: 1205.3453 [INSPIRE].

[62] J.M. Campbell and R.K. Ellis, An update on vector boson pair production at hadron colliders, Phys. Rev. D 60 (1999) 113006 [hep-ph/9905386] [InSPIRE].

[63] J.M. Campbell, R.K. Ellis and C. Williams, Vector boson pair production at the LHC, JHEP 07 (2011) 018 [arXiv: 1105.0020] [INSPIRE].

[64] J.M. Campbell and R.K. Ellis, $t \bar{t} W^{+-}$production and decay at NLO, JHEP 07 (2012) 052 [arXiv: 1204.5678] [INSPIRE].

[65] ATLAS collaboration, Search for exotic same-sign dilepton signatures ( $b^{\prime}$ quark, $T_{5 / 3}$ and four top quarks production) in $4.7 \mathrm{fb}^{-1}$ of pp collisions at $\sqrt{s}=7 \mathrm{TeV}$ with the ATLAS detector, ATLAS-CONF-2012-130, CERN, Geneva Switzerland (2012).

[66] ATLAS collaboration, A search for $t \bar{t}$ resonances with the ATLAS detector in $2.05 \mathrm{fb}^{-1}$ of proton-proton collisions at $\sqrt{s}=7 \mathrm{TeV}$, Eur. Phys. J. C 72 (2012) 2083 [arXiv:1205.5371] [INSPIRE].

[67] A.L. Read, Presentation of search results: the $C L_{s}$ technique, J. Phys. G 28 (2002) 2693 [INSPIRE].

[68] McLimit website - reference for the McLimit software, http://www-cdf.fnal.gov/ trj/mclimit/production/mclimit.html. 